\title{
On Resonant Classical Hamiltonians with Two Equal Frequencies
}

Martin Kummer

Department of Mathematics, The University of Toledo, Toledo, Ohio 43606, USA

Abstract. This paper contains a detailed study of the flow that the classical Hamiltonian

$$
H=\frac{1}{2}\left(x_{1}^{2}+y_{1}^{2}\right)-\frac{1}{2}\left(x_{2}^{2}+y_{2}^{2}\right)+\mathcal{O}_{3}
$$

induces near the origin of its phase space $R^{4}$. Here the perturbation term $\mathcal{O}_{3}$ represents a convergent power series. In particular, criteria for the existence and stability of periodic orbits are developed and expressed in terms of canonical invariants that are extracted from the perturbation term.

\section{Introduction}

The present work is dedicated to a study of the flow that the Hamiltonian (1.1) induces near the origin of its phase space $R^{4}$.

The methods that we will apply in our study are essentially the same as those of [1]. In that paper a Hamiltonian was studied which can be obtained from the Hamiltonian (1.1) of the present work by replacing the minus sign in the leading term

$$
L=\frac{1}{2}\left(x_{1}^{2}+y_{1}^{2}\right)-\frac{1}{2}\left(x_{2}^{2}+y_{2}^{2}\right)
$$

by a plus sign. The symplectic transformations that leave the leading term $L$ invariant constitute the group $U(1,1)$, and correspondingly the Gustavson normal form of our Hamiltonian is best viewed as a function of a canonical set: $M_{0}=\frac{L}{2}$, $M_{1}, M_{2}, M_{3}$ of generators of that group, that is to say, as a function over the Liealgebra $u(1,1)$. Here $L$ or $M_{0}$ generates the center of the group, whereas $M_{1}, M_{2}, M_{3}$ generate $\mathrm{SU}(1,1)$.

We bring the Hamiltonian (1.1) into normal form up to order $2 n$, where $n$ is determined by the condition that the lowest degree, non-trivial polynomial $K_{n}$ in the generators of the group $U(1,1)$ that appears in the normal form is homogeneous of degree $n$. Here, a polynomial is called non-trivial if it is not just a function of $M_{0}=\frac{L}{2}$ 
alone. Accordingly, after this transformation our Hamiltonian consists of a part which is in normal form and can be written as a sum of $K_{n}$ plus a polynomial $P\left(M_{0}\right)$ of degree $n-1$ in $M_{0}$ (beginning with a term $2 M_{0}$ ), and a remainder $\mathcal{O}_{2 n+1}$ which is not in normal form. We will refer to the former as the unperturbed or truncated Hamiltonian and to the latter as the perturbation.

The manifold that is obtained by identifying points of the same orbit that $P\left(M_{0}\right)$ induces on a manifold $M_{0}=$ const, is shown to be a two-sheeted hyperboloid: $h^{2}$ imbedded in the Lie-algebra su(1,1), the latter being identified with $R^{3}$. According to a theorem by Moser [2] the non-degenerate critical points of $K_{n}$ on that orbitmanifold give rise to periodic orbits of the unperturbed Hamiltonian. These periodic orbits are stable/unstable, precisely if the critical points (c.p.), which give rise to them, are stable/unstable. They can be continued to periodic orbits of the full Hamiltonian, provided that the absolute value of the energy is kept sufficiently small. Unstable periodic orbits of the truncated Hamiltonian are continued to unstable orbits of the full Hamiltonian. However, in order that a stable periodic orbit of the truncated Hamiltonian remain stable after its continuation, a certain non-linear canonical invariant has to be non-zero [see Theorem 2 and Expression (3.8)]. This follows from a suitable application of Moser's twist theorem [3].

An essential difference between the Hamiltonian of the present work and the one studied in [1] is that the leading term in the latter Hamiltonian is positive definite, while that in the Hamiltonian (1.1) is indefinite. Thus, the stability of the origin of $R^{4}$ is no longer guaranteed. Criteria that allow a determination of the nature of this equilibrium point were developed by Sokol'skii [4]. In Section 4 we generalize those criteria to Hamiltonians of the form (1.23) (see Theorem 3).

In Sections 5 and 6 we confine ourselves to the case $n=2$. For this special case we present a complete classification of all periodic orbits that arise from nondegenerate critical points of $K^{(2)}$ on the orbit manifold $h^{2}$ in terms of non-linear canonical invariants which are extracted from the polynomial $K^{(2)}$. For the general theory underlying this approach to periodic solutions see [2]. The coefficients of $K^{(2)}$ are represented by a vector $\boldsymbol{b}$ and a symmetric 2 by 2 matrix $\mathfrak{A}$. The latter represents a quadratic form over the vector space $\operatorname{su}(1,1) \approx R^{3}$ and because the group $\mathrm{SU}(1,1)$ induces restricted Lorentz-transformations [i.e. elements of $\left.\mathrm{SO}^{\uparrow}(2,1)\right]$ in this vector space the question naturally arises whether the matrix $\mathfrak{A}$ can be diagonalized by a transformation of the group $\mathrm{SO}^{\uparrow}(2,1)$. (See Theorem 4.) Depending on whether this is possible or not we distinguish the two main Cases I and II which in turn decompose into several subcases. In the Case I the classification scheme of critical point bears great resemblance to the one developed in [1].

However, the Case II has no counterpart in the treatment of the Hamiltonian of [1]. In this case our classification scheme is based on a (real) normal form of $\mathfrak{A}$ which differs from diagonal form. (See Theorem 7 and for the generic subcase of Case II the end of Section 6.)

Applying Sokol'skii's criteria to the generic subcase of Case I we see that the origin of $R^{4}$ is stable/unstable precisely if the total index of all c.p. that $K^{(2)}$ possesses on $h^{2}$ is $+2 /-2$. (See Theorems 5 and 6.)

Also, in some subcases of Case II, a connection between the total index and the stability of the origin of $R^{4}$ can be exhibited (see e.g. Theorem 8). In Section 7 we 
illustrate Case I with help of an example that bears great resemblance to the model of Henon-Heiles [6].

Finally, we remark that the methods of this paper and [1] could also be applied to reasonant Hamiltonians with two degrees of freedom for which the ratio of the frequencies is different from one. However, these cases have been studied almost exhaustively by other authors (see e.g. [8]).

\section{General Considerations and Preparations}

The goal of this section is to collect as much general information as possible about the flow that the classical Hamiltonian

$$
H\left(x_{1}, y_{1}, x_{2}, y_{2}\right)=\frac{1}{2}\left(x_{1}^{2}+y_{1}^{2}\right)-\frac{1}{2}\left(x_{2}^{2}+y_{2}^{2}\right)+\sum_{k=3}^{\infty} H_{k}\left(x_{1}, y_{1}, x_{2}, y_{2}\right)
$$

induces in its phase space $R^{4}$. Here, $H_{k}$ is a homogeneous polynomial of degree $k$ over $R^{4}$ and the infinite sum is convergent in some complex neighborhood of the origin of $R^{4}$. The vector field $X_{H}$ in $R^{4}$, associated with the Hamiltonian (1), is the unique vector field whose inner product with the 2 -form

$$
\omega=\sum_{k=1}^{2}\left(d y_{k} \wedge d x_{k}\right)
$$

yields $-d H$, in formulae:

$$
\left.X_{H}\right\lrcorner \omega=-d H \text {. }
$$

The group of linear canonical transformations leaving the leading term of our Hamiltonian invariant is $U(1,1)$. This is most easily seen if the variables

$$
z_{k}=2^{-1 / 2}\left(x_{k}+i y_{k}\right) \quad(k=1,2)
$$

and the sesquilinear expressions

$$
M_{k}=\frac{1}{2} Z^{\dagger} \sigma_{k} Z, \quad k=0,1,2,3
$$

are introduced. Here $Z=\left(\begin{array}{l}z_{1} \\ z_{2}\end{array}\right), Z^{\dagger}=\left(\bar{z}_{1}, z_{2}\right)$ and the $\sigma_{k}$ 's $(k=0,1,2,3)$ are the Paulimatrices, where for notational convenience we have taken the liberty to call $\sigma_{0}$ what is conventionally called $\sigma_{3}$ and vice versa, i.e. we define:

$$
\sigma_{0}=\left(\begin{array}{rr}
1 & 0 \\
0 & -1
\end{array}\right), \quad \sigma_{1}=\left(\begin{array}{ll}
0 & 1 \\
1 & 0
\end{array}\right), \quad \sigma_{2}=\left(\begin{array}{rr}
0 & -i \\
i & 0
\end{array}\right), \quad \sigma_{3}=\left(\begin{array}{ll}
1 & 0 \\
0 & 1
\end{array}\right) .
$$

If expressed in the $z_{k}$-variables, the leading term of the Hamiltonian (1) simply becomes $2 M_{0}$ and the 2 -form $\omega$ is given by the following expression:

$$
\omega=\frac{1}{i} \sum_{k=1}^{2}\left(d z_{k} \wedge d \bar{z}_{k}\right)=i d\left(Z^{\dagger} \sigma_{0} d Z\right)
$$

The new coordinates make it apparent that the group of linear transformations leaving $\omega$ and $M_{0}$ invariant is

$$
U(1,1)=\left\{U \in \mathrm{GL}(2) \mid U^{\dagger} \sigma_{0} U=\sigma_{0}\right\},
$$

where GL(2) is the group of complex (invertible) two by two matrices. 
The $M_{k}$ 's $(k=0,1,2,3)$ as defined in (5) are nothing but the infinitesimal generators of the group $U(1,1)$ and accordingly they satisfy the bracket relations

$$
\begin{array}{ll}
{\left[M_{3}, M_{1}\right]=i M_{2},} & {\left[M_{2}, M_{3}\right]=i M_{1}} \\
{\left[M_{1}, M_{2}\right]=-i M_{3},} & {\left[M_{0}, M_{k}\right]=0 \quad(k=1,2,3)}
\end{array}
$$

with respect to the Poisson-bracket

$$
[f, g]=\sum_{k=1}^{2}\left(\frac{\partial f}{\partial z_{k}} \frac{\partial g}{\partial \bar{z}_{k}}-\frac{\partial g}{\partial z_{k}} \frac{\partial f}{\partial \bar{z}_{k}}\right)
$$

which is associated with the 2 -form $i \omega$. They are not functionally independent but satisfy the relation:

$$
M_{3}^{2}-M_{1}^{2}-M_{2}^{2}=M_{0}^{2} \text {. }
$$

By associating with each point $\left(x_{1}, y_{1}, x_{2}, y_{2}\right)$ of $R^{4}$ the point

$$
x=\frac{M_{1}}{M_{0}} \quad y=\frac{M_{2}}{M_{0}} \quad z=\frac{M_{3}}{M_{0}}
$$

of $R^{3}$ we obtain a map $\Pi: R^{4} \rightarrow R^{3}$, which is defined everywhere except on the cone:

$$
M_{0}=0 \text {. }
$$

It maps each hyperboloid $h_{m}^{3}: M_{0}=m$ onto the upper sheet $h_{+}^{2}(z \geqq 1)$ or lower sheet $h_{-}^{2}(z \leqq-1)$ of the two dimensional hyperboloid $h^{2}: h(x)=1$, where [see (11)]

$$
h(x)=-x^{2}-y^{2}+z^{2}
$$

depending on whether $m$ is positive or negative.

The meaning of the mapping $\Pi$ and the hyperboloid $h^{2}$ becomes more transparent if we introduce a new symplectic chart in the region $\left|z_{1}\right|^{2}>0$ of $R^{4}$ by means of the following succession of transformations. We set:

$$
N_{k}=\left|z_{k}\right|^{2}, \quad z_{k}=N_{k}^{1 / 2} e^{-i \alpha_{k}} \quad(k=1,2)
$$

and

$$
L=N_{1}-N_{2}=2 M_{0}, \quad R=N_{2}, \quad \phi=\alpha_{1}+\alpha_{2}
$$

and finally

$$
\xi=\sqrt{2 R} \cos \phi, \quad \eta=\sqrt{2 R} \sin \phi .
$$

We find:

$$
\omega=d N_{1} \wedge d \alpha_{1}+d N_{2} \wedge d \alpha_{2}=d L \wedge d \alpha_{1}+d R \wedge d \phi=d L \wedge d \alpha_{1}+d \xi \wedge d \eta
$$

i.e., $L, \alpha_{1}, \xi, \eta$ are the coordinates of a symplectic chart covering the region $N_{1}>0$ (hence in particular the region $M_{0}>0$ ) of $R^{4}$. They relate to the original variables $\left(x_{1}, y_{1}, x_{2}, y_{2}\right)$ as follows:

$$
\begin{aligned}
& x_{1}=\left(2 L+\xi^{2}+\eta^{2}\right)^{1 / 2} \cos \alpha_{1} \\
& y_{1}=-\left(2 L+\xi^{2}+\eta^{2}\right)^{1 / 2} \sin \alpha_{1} \\
& x_{2}=\xi \cos \alpha_{1}+\eta \sin \alpha_{1} \\
& y_{2}=\xi \sin \alpha_{1}-\eta \cos \alpha_{1} .
\end{aligned}
$$


The transformation (19) shows that each hyperboloid $h_{m}^{3}(m>0)$ is analytically diffeomorphic to $R^{2} \times S^{1}$ and therefore also to $h_{+}^{2} \times S^{1}$ (because $h_{+}^{2}$ is obviously diffeomorphic to the plane).

These considerations show that the map $\Pi$ defined in (12) is nothing but the projection of a trivial fiber-bundle

$$
\Pi: h_{m}^{3} \cong h_{+}^{2} \times S^{1} \rightarrow h_{+}^{2}
$$

whose fibers are the orbits of the Hamiltonian $M_{0}$ (or $L$ ) on the surface $h_{m}^{3}$. In other words, $h_{+}^{2}$ is the orbit manifold associated with the flow that $M_{0}$ induces on each surface $h_{m}(m>0)$ and $\Pi$ is the canonical map that identifies points lying on the same oriented orbit. In the new chart the map $\Pi: R^{4}-\left\{M_{0}=0\right\} \rightarrow h^{2} \subset R^{3}$ has the following expression:

$$
\begin{aligned}
& \Pi:\left(L, \alpha_{1}, \xi, \eta\right) \rightarrow x \\
& =\frac{1}{L}\left\{\left(2 L+\xi^{2}+\eta^{2}\right)^{1 / 2} \xi,\left(2 L+\xi^{2}+\eta^{2}\right)^{1 / 2} \eta, L+\xi^{2}+\eta^{2}\right\} .
\end{aligned}
$$

Its restriction to the hyperboloid $h_{m}^{3}(m>0)$ is obtained by setting: $L=2 m$. Finally, we note that if $\Pi$ is restricted to the surface

$$
h_{m}^{3} \cap\left\{\alpha_{1}=\alpha_{1}^{0} \equiv \text { const }\right\} \quad(m>0)
$$

it becomes a diffeomorphism with the inverse

$$
\xi=\mathrm{x}\left(\frac{2 m}{z+1}\right)^{1 / 2} \quad \eta=y\left(\frac{2 m}{z+1}\right)^{1 / 2} .
$$

That is to say, the flow of $M_{0}$ on the hyperboloid $h_{m}^{3}(m>0)$ possesses transversal sections that are diffeomorphic to the orbit manifold $h_{+}^{3}$. This result will be of crucial importance when we discuss periodic orbits of our Hamiltonian in Sections 2 and 3.

As a consequence of the Birkhoff-Gustavson normal form theorem, our Hamiltonian can be brought into the form

$$
H=P\left(M_{0}\right)+K_{n}\left(M_{0}, M\right)+\mathcal{O}_{2 n+1}
$$

by means of a real analytic canonical transformation.

Here, $\boldsymbol{M}=\left(M_{1}, M_{2}, M_{3}\right), P\left(M_{0}\right)=2 M_{0}+\ldots$ is a polynomial of degree $\leqq n-1$ in $M_{0}$ whose lowest order term is $2 M_{0}$, and $K_{n}\left(M_{0}, M\right)$ denotes a homogeneous polynomial of degree $n$ in the variables $\left(M_{0}, \boldsymbol{M}\right)$ that, on account of relation (11) is only determined up to an additive polynomial belonging to the ideal generated in the algebra of all polynomials in the variables $\left(M_{0}, \boldsymbol{M}\right)$ by the quadratic polynomial

$$
M_{1}^{2}+M_{2}^{2}-M_{3}^{2}+M_{0}^{2} .
$$

The integer $n$ is determined by the condition that (modulo a polynomial in this ideal) $K_{n}\left(M_{0}, \boldsymbol{M}\right)$ is not just a polynomial of $M_{0}$ alone. Thus, $K_{n}\left(M_{0}, \boldsymbol{M}\right)$ can be assumed to possess a representation

$$
K_{n}\left(M_{0}, M\right)=\sum_{j=0}^{n} M_{0}^{j} K_{n j}(M)
$$

in which at least one of the polynomials $K_{n j}(\boldsymbol{M})$ of degree $n-j$ in the variables $\boldsymbol{M}$ $(j=0,1, \ldots, n-1)$ is neither zero nor divisible by $M_{1}^{2}+M_{2}^{2}-M_{3}^{2}$. In this connection, 
we note for later use that if $a_{k_{0} k_{1} k_{2} k_{3}}$ is the coefficient of $M_{0}^{k_{0}} M_{1}^{k_{1}} M_{2}^{k_{2}} M_{3}^{k_{3}}$ in $K_{n}$ and $c_{k_{0} k_{1} k_{2} k_{3}}$ the corresponding coefficient in a polynomial of the expression (24), $K_{n}$ is unaltered by a simultaneous translation

$$
a_{k_{0} k_{1} k_{2} k_{3}} \rightarrow a_{k_{0} k_{1} k_{2} k_{3}}+c_{k_{0} k_{1} k_{2} k_{3}}
$$

of its coefficients.

The symbol $\mathcal{O}_{2 n+1}$ in (23) represents a convergent power series in the variables $x_{1}, y_{1}, x_{2}, y_{2}$ that begins with a term of order $2 n+1$. The most important case is the case in which $n=2$. Sections 5 and 6 of our present work will almost entirely de dedicated to a detailed analysis of this special case.

There is another even more important reason why the coefficients of the polynomial $K_{n}$ (which are polynomials in the coefficients of $H_{3}, \ldots, H_{2 n}$ ) are not uniquely determined.

Namely, any transformation of the $z_{k}$-variables $(k=1,2)$ of the form

$$
\hat{Z}=U Z, \quad U \in \operatorname{SU}(1,1) \text {, }
$$

where $\mathrm{SU}(1,1)=\left\{U=\left(\begin{array}{ll}a & b \\ \bar{b} & \bar{a}\end{array}\right) ; a, b \in \mathbb{C},|a|^{2}-|b|^{2}=1\right\}$ is canonical and induces a transformation $O(U) \in \mathrm{SO}(2,1)$ of the vector $\boldsymbol{M}$ by means of the formula:

$$
U^{\dagger}(\boldsymbol{\sigma} \cdot \boldsymbol{M}) U=\boldsymbol{\sigma} \cdot \hat{\boldsymbol{M}}, \hat{\boldsymbol{M}}=O(U) \boldsymbol{M},
$$

where $\boldsymbol{\sigma}=\left(\sigma_{1}, \sigma_{2}, \sigma_{3}\right)$ is the vector of Pauli matrices. It is well known that the image of the map

$$
O: \mathrm{SU}(1,1) \rightarrow \mathrm{SO}(2,1),
$$

defined in this way, is precisely the subgroup $\mathrm{SO}^{\uparrow}(2,1)$ of $\mathrm{SO}(2,1)$, characterized by the condition

$$
\mathrm{SO}^{\uparrow}(2,1)=\left\{O=\left(o_{i k}\right) \in \mathrm{SO}(2,1) \mid o_{33} \geqq 1\right\} .
$$

Of the two connected components that the group $\operatorname{SO}(2,1)$ possesses the one containing the unit element coincides precisely with the subgroup $\operatorname{SO}^{\uparrow}(2,1)$. The transformations of $\mathrm{SO}^{\uparrow}(2,1)$ in turn induce transformations in the space of homogeneous polynomials of which $K_{n}$ is a member. This fact represents the major reason for the non-uniqueness of $K_{n}$ and will play an important role in the sequel.

Finally, we note that the transformation

$$
t \rightarrow-t, \quad z_{1} \leftrightarrow z_{2}
$$

replaces $K_{n}\left(M_{0}, \boldsymbol{M}\right)$ by $-K_{n}\left(-M_{0}, \boldsymbol{M}\right)$. In the $\boldsymbol{x}$-space this transformation induces a reflection

$$
\boldsymbol{x} \rightarrow-\boldsymbol{x} .
$$

This is most easily seen from the fact that

$$
\boldsymbol{M}=\left(\operatorname{Re}\left(\bar{z}_{1} \bar{z}_{2}\right), \operatorname{Im}\left(\bar{z}_{1} \bar{z}_{2}\right), \frac{N_{1}+N_{2}}{2}\right)
$$

is invariant under the transformation (30), whereas $M_{0}=\frac{1}{2}\left(N_{1}-N_{2}\right)$ changes its sign.

In the following we shall refer to the transformation (30) as time reversal. 


\section{The Flow of the Truncated Hamiltonian}

In this section we shall drop the term $\mathcal{O}_{2 n+1}$ in (1.23) and call the resulting Hamiltonian $H_{0}$ the truncated or unperturbed Hamiltonian. We intend to gather as much qualitative information about its flow in $R^{4}$ as possible. In particular, we are interested in its periodic orbits.

First, we note that the flow of $H_{0}$ carries each hyperboloid $h_{m}^{3}(m \neq 0)$ into itself and that the corresponding flow on $h^{2}$ is the restriction to $h^{2}$ of the flow in $R^{3}$, governed by the differential equations

$$
\frac{d \boldsymbol{x}}{d \tau}=\frac{1}{2}\left(\nabla h \times \nabla K^{(n)}\right)(\boldsymbol{x}),
$$

the last statement being an immediate consequence of the bracket relations (1.9). The function $K^{(n)}$ appearing in (1) is related to the function $K_{n}$, defined in (1.23), by $K^{(n)}(\boldsymbol{x})=K_{n}(1, \boldsymbol{x})$ and $\tau$ is a new time-parameter, given by $\tau=M_{0}^{n-1} t$.

The flow induced by $K^{(n)}$ on $h^{2}$ determines the flow of $H_{0}$ on each hyperboloid $h_{m}^{3}$ completely. Indeed, if $\boldsymbol{x}(\tau)$ is a solution of (1) then, for $m>0, \xi$ and $\eta$ can be obtained as functions of $t$ from the expressions (1.22). Furthermore, from (1.20) we infer

$$
\frac{\partial \boldsymbol{x}}{\partial L}=\frac{1}{L(z+1)}(\boldsymbol{k}-z \boldsymbol{x}),
$$

where $\boldsymbol{k}=(0,0,1)$ is the unit vector in the positive $z$-direction. Hence, on $h_{m}^{3}(m>0)$ the rate of change of $\alpha_{1}$ with respect to $t$ is

$$
\frac{d \alpha_{1}}{d t}=\frac{\partial H_{0}}{\partial L}=\frac{1}{2} P^{\prime}(m)+\frac{m^{n-1}}{2}\left[n K^{(n)}(\boldsymbol{x})+\frac{1}{z+1} \nabla K^{(n)}(\boldsymbol{x}) \cdot(\boldsymbol{k}-z \boldsymbol{x})\right] .
$$

A simple integration of the last equation yields $\alpha_{1}$ as a function of $t$.

We proceed therefore to study the flow induced by the differential Equation (1) on $h^{2}$. It is clear that the orbits of this flow are obtained by intersecting the level surfaces of $K^{(n)}$ in $R^{3}$ with $h^{2}$. In particular, the critical points (abbreviated: c.p.) of $K^{(n)}$ on $h^{2}$ are the equilibrium points of the flow. They are points $e \in R^{3}$ satisfying $h(\boldsymbol{e})=1$ and

$$
\nabla K^{(n)}(e)=\frac{\lambda}{2} \nabla h(e)
$$

with some number $\lambda \in R$ which we shall call the multiplier of $e$. They correspond to periodic orbits of $H_{0}$ in $R^{4}$. Indeed, to a c.p. $e$ of $K^{(n)}$ on $h_{+}^{2}$ the relations (1.14) and (3) yield a family of periodic orbits, one on each surface $h_{m}^{3}(m>0)$, with frequency

$$
\frac{1}{2} P^{\prime}(m)+\frac{m^{n-1}}{2} n K^{(n)}(e)
$$

As pointed out in Section 1, the surfaces

$$
h_{m}^{3} \cap\left\{\alpha_{1}=\alpha_{1}^{0} \equiv \text { const }\right\}
$$

are diffeomorphic to $h_{+}^{2}$. Because they are transversal sections to the periodic orbits associated with the c.p. $e$ on $h^{2}$ it follows that each member of the family of periodic orbits is stable/unstable, precisely if the c.p. $\boldsymbol{e}$ is stable/unstable. 
In order to investigate the nature of a c.p. we let $\mathfrak{A}(e)$ denote the Hessian of $K^{(n)}$, evaluated at $\boldsymbol{e}$ and we introduce the "Lorentzian" scalar product:

$$
\langle\boldsymbol{x}, \boldsymbol{y}\rangle=(\boldsymbol{x}, G \boldsymbol{y})
$$

in $R^{3}$. Here, (,) denotes the usual Euclidian inner product of $R^{3}$ and $G$ is the 3 by 3 matrix

$$
G=\operatorname{diag}(-1,-1,1) .
$$

Clearly, the quadratic form, associated with the scalar product (9) agrees with the function $h(\boldsymbol{x})$, defined in (1.14).

It is not difficult to see that a c.p. $e$ of $K^{(n)}$ on $h^{2}$ is stable or unstable depending on whether the restriction of the quadratic form

$$
Q(y)=\lambda h(y)-(y, \mathfrak{Y}(e) y)=(y,[\lambda G-\mathfrak{A}(e)] y)
$$

to the tangent plane $T(\boldsymbol{e})$ to $h^{2}$ at $e$ is definite or indefinite. If it is semi-definite the c.p. is called degenerate. Unless stated otherwise, c.p. in the sequel will always mean : non-degenerate c.p.

Our criterion translates into the following formula for the index of the c.p. $e$ :

$$
\text { ind } \boldsymbol{e}=\operatorname{sgn} \Delta(\boldsymbol{e}) \text {, }
$$

where

$$
\Delta(e)=-\operatorname{det}\left(\begin{array}{cc}
\lambda G-\mathfrak{A}(e) & G e \\
(G e)^{T} & 0
\end{array}\right)=\langle e, \operatorname{adj}(\lambda-G \mathfrak{A}(e)) e\rangle
$$

and $\Delta(\boldsymbol{e}) \neq 0$ as a result of our non-degeneracy assumption ( $\mathrm{adj}=$ algebraic adjoint).

In most instances we have limited our discussion to the region $M_{0}>0$ of $R^{4}$ and correspondingly to the flow of $K^{(n)}$ on $h_{+}^{2}$. We do not lose any generality as a result of this limitation because, as the following theorem shows, upper and lower sheet of the hyperboloid $h^{2}$ are interchanged by the time reversal (1.30).

Theorem 1. The flow induced by $K^{(n)}$ (via the differential Eq. (1)) on $h_{\gamma}^{2}(\gamma= \pm 1)$ is mapped by the time reversal (1.30) onto the time-reversed flow $(\tau \rightarrow-\tau)$, induced by $\tilde{K}^{(n)}(\boldsymbol{x}) \equiv K^{(n)}(-\boldsymbol{x})$ on $h_{-\gamma}^{2}$. In particular, if e is a c.p. of $K^{(n)}$ on $h_{\gamma}^{2}$ with multiplier $\lambda$ (see (4)), - $e$ is a c.p. of $\tilde{K}^{(n)}$ on $h_{-\gamma}^{2}$ with the same multiplier and the two c.p. have the same index.

We leave the trivial proof to the reader.

\section{Periodic Solutions of the Full Hamiltonian}

The families of periodic solutions of the truncated Hamiltonian that we found in the last section can be continued to periodic solutions of the full Hamiltonian (1.23), provided that the absolute value of the energy is kept sufficiently small. The possibility of their continuation follows immediately from a theorem of Moser ([2, Theorem 3, p. 624]). However, the question remains whether a stable orbit of $H_{0}$ will remain stable in the process of this continuation. In order to investigate this question we first remark that, by a suitable transformation $U \in \mathrm{SU}(1,1)$ of the 
$z_{k}$-variables $(k=1,2)$, preceded, if necessary, by a time reversal (1.30) it is possible to bring any c.p. lying on $h^{2}$ into the position $\boldsymbol{k}$ [see Formulae (1.27) and (1.28)]. Moreover, it is easy to see that $U$ can be chosen in such a way that the 12 element in the Hessian $\mathfrak{U}(\boldsymbol{k})=\left(A_{i k}\right)_{1 \leqq i, k \leqq 3}$ vanishes

$$
A_{12}=0 \text {. }
$$

The Equations (2.4), with $\boldsymbol{e}$ replaced by $\boldsymbol{k}$, yield

$$
\frac{\partial K^{(n)}}{\partial x}(\boldsymbol{k})=\frac{\partial K^{(n)}}{\partial y}(\boldsymbol{k})=0, \quad \frac{\partial K^{(n)}}{\partial z}(\boldsymbol{k})=\lambda,
$$

and the condition (2.10) for the stability of the c.p. $\boldsymbol{k}$ simply becomes

$$
\Delta(\boldsymbol{k})=A B>0,
$$

where $A=\lambda+A_{11}, B=\lambda+A_{22}$. In order to be able to formulate a sufficient stability condition for the continued family of periodic solutions corresponding to the c.p. $\boldsymbol{k}$ we need the coefficients in the expansion of the function

$$
F(\xi, \eta)=K^{(n)}(x(2, \xi, \eta))-K^{(n)}(\boldsymbol{k})
$$

about the point $\xi=\eta=0$. Here, $\boldsymbol{x}(2, \xi, \eta)$ denotes the right side of (1.20), where $L$ has been given the value 2 . We find

$$
F(\xi, \eta)=\frac{1}{2}\left(A \xi^{2}+B \eta^{2}\right)+\sum_{k=0}^{3} B_{k 3-k} \xi^{k} \eta^{3-k}+\sum_{k=0}^{4} C_{k 4-k} \xi^{k} \eta^{4-k}+\mathcal{O}_{5}
$$

where $\mathcal{O}_{5}$ is a convergent power series in $\xi, \eta$, beginning with a term of order five. If we use the notation $C=\lambda-A_{33}$ and

$$
A_{113}=\frac{\partial^{3} K^{(n)}}{\partial x^{2} \partial z}(k), \text { etc. }
$$

the coefficients in the expansion (5) are given by the following expressions

$$
\begin{aligned}
& B_{30}=\frac{1}{2} A_{13}+\frac{1}{6} A_{111}, \quad B_{03}=\frac{1}{2} A_{23}+\frac{1}{6} A_{222}, \\
& B_{12}=\frac{1}{2}\left(A_{13}+A_{122}\right), \quad B_{21}=\frac{1}{2}\left(A_{23}+A_{112}\right) . \\
& C_{40}=\frac{1}{8}(A-C)+\frac{1}{4} A_{113}+\frac{1}{24} A_{1111}, \\
& C_{04}=\frac{1}{8}(B-C)+\frac{1}{4} A_{223}+\frac{1}{24} A_{2222}, \\
& C_{22}=\frac{1}{8}(A+B-2 C)+\frac{1}{4}\left(A_{113}+A_{223}+A_{1122}\right) .
\end{aligned}
$$

The expressions for the coefficients $C_{13}$ and $C_{31}$ have been omitted because they are not needed in the sequel.

With these preparation behind us, we are in the position to formulate a theorem which relates the stability of our c.p. $\boldsymbol{k}$ to the stability of the associated family of periodic orbits of the full Hamiltonian:

Theorem 2. (i) To each unstable c.p. of $K^{(n)}$ on $h^{2}$ there corresponds an unstable oneparameter family of periodic orbits of the full Hamiltonian, the family parameter being the energy, which we think of being kept sufficiently small. 
(ii) An analogous statement holds if the c.p. is stable provided that in addition (after transformation of the c.p. into $\boldsymbol{k}$ ) the following expression is non-zero:

$$
\begin{aligned}
& \frac{3}{2} A\left[4 A^{2} B_{03}^{2}+\left(B_{03} A+B_{21} B\right)^{2}\right]+\frac{3}{2} B\left[4 B^{2} B_{30}^{2}+\left(B_{30} B+B_{12} A\right)^{2}\right] \\
& \quad-A B\left(3 C_{40} B^{2}+C_{22} A B+3 C_{04} A^{2}\right) .
\end{aligned}
$$

Remark. The expression (8) simplifies considerably if all A's with three and four subscripts in the list (7) vanish as is the case if $n=2$. In this instance the expression (8) after multiplication with the factor eight becomes

$$
12\left(B^{3} A_{13}^{2}+A^{3} A_{23}^{2}\right)-4(A B)^{2} T+3(A+B)^{2} D,
$$

where $T=\operatorname{Trace}(\lambda-G \mathfrak{U}(\boldsymbol{k})), D=\operatorname{det}(\lambda-G \mathfrak{U}(\boldsymbol{k}))$.

The proof of Theorem 2 proceeds along the lines of Theorem 1 of [1]. We shall only sketch the proof of statement (ii).

Setting $z_{k}=\varepsilon \hat{z}_{k}(k=1,2)$, where $\varepsilon$ is a small positive number and using the chart $L$, $\alpha_{1}, \xi, \eta[$ see (1.16) to (1.18)] we have:

$$
\begin{aligned}
& H=\varepsilon^{2} \hat{H} \quad L=\varepsilon^{2} \hat{L}, \quad \xi=\varepsilon \hat{\xi} \quad \eta=\varepsilon \hat{\eta} \\
& \hat{H}=\varepsilon^{-2} P\left(\frac{\varepsilon^{2} L}{2}\right)+\varepsilon^{2(n-1)} K^{(n)}(x(L, \xi, \eta))+\mathcal{O}\left(\varepsilon^{2 n-1}\right),
\end{aligned}
$$

where the symbol $\mathcal{O}\left(\varepsilon^{2 n-1}\right)$ denotes a function that is real analytic in the variables $L$, $\alpha_{1}, \xi, \eta, \varepsilon$ at the point $\left(L, \alpha_{1}, 0,0,0\right)(L>0)$, periodic in $\alpha_{1}$ with period $2 \pi$ and which begins with a term of order $2 n-1$ in $\varepsilon$.

On the surface $\hat{H}=2$, relation (11) implicitly defines $-L$ as a function of $\alpha_{1}, \xi, \eta$, $\varepsilon$, which is real analytic at $\left(\alpha_{1}, 0,0,0\right)$, periodic in $\alpha_{1}$ with period $2 \pi$ and which takes the value two for $\varepsilon=0$. Subtracting its value at $\left(\alpha_{1}, 0,0, \varepsilon\right)$ we obtain a function $\Lambda$ whose power series in $\varepsilon$ begins as follows

$$
\Lambda\left(\alpha_{1}, \xi, \eta, \varepsilon\right)=\varepsilon^{2(n-1)}[F(\xi, \eta)+\mathcal{O}(\varepsilon)] .
$$

The function $\Lambda$ together with the 2 -form

$$
d \xi \wedge d \eta
$$

determines the flow on the energy surface $H=2 \varepsilon^{2}, \alpha_{1}$ serving as new time parameter. Introducing complex normal coordinates $\zeta, \bar{\zeta}$ in the lowest order term of (12) the transformed Hamiltonian becomes

$$
\hat{\Lambda}\left(\alpha_{1}, \zeta, \bar{\zeta}, \varepsilon\right)=\varepsilon^{2(n-1)}[\hat{F}(\varrho)+\mathcal{O}(\varepsilon)]
$$

where $\varrho=\zeta \bar{\zeta}$ and $\hat{F}$ is the normal form of $F$. Because in the new coordinates the twoform (13) becomes $i(d \zeta \wedge d \bar{\zeta})$, the equations associated with the Hamiltonian $\hat{\Lambda}$ are

$$
\begin{aligned}
& \dot{\zeta}=i \varepsilon^{2(n-1)}\left[\hat{F}^{\prime}(\varrho) \zeta+\mathcal{O}(\varepsilon)\right] \\
& \dot{\bar{\zeta}}=-i \varepsilon^{2(n-1)}\left[\hat{F}^{\prime}(\varrho) \bar{\zeta}+\mathcal{O}(\varepsilon)\right],
\end{aligned}
$$

where the dot denotes differentiation with respect to $\alpha_{1}$. An explicit calculation of $\hat{F}(\varrho)$ yields

$$
\hat{F}(\varrho)=v \varrho+\kappa \varrho^{2}+\mathcal{O}\left(\varrho^{3}\right),
$$


where $v=(\Delta)^{1 / 2} \operatorname{sgn} A, \kappa=-\frac{1}{2 \Delta^{2}}$ expression (8) and $\Delta=A B>0$ as a consequence of our assumption that the c.p. $\boldsymbol{k}$ is stable [see (3)]. If we follow the trajectories from $\alpha_{1}=0$ to $\alpha_{1}=2 \pi$ we obtain a measurepreserving map

$$
M: \begin{aligned}
& \zeta_{1}=\zeta+2 \pi i \varepsilon^{2(n-1)}\left[\hat{F}^{\prime}(\varrho) \zeta+\mathcal{O}(\varepsilon)\right] \\
& \bar{\zeta}_{1}=\bar{\zeta}-2 \pi i \varepsilon^{2(n-1)}\left[\hat{F}^{\prime}(\varrho) \bar{\zeta}+\mathcal{O}(\varepsilon)\right]
\end{aligned}
$$

where the term $\mathcal{O}(\varepsilon)$ no longer depends on $\alpha_{1}$. Obviously, for $\varepsilon=0$ the function

$$
f(\zeta, \bar{\zeta}, \varepsilon)=\hat{F}^{\prime}(\varrho) \zeta+\mathcal{O}(\varepsilon)
$$

has an isolated zero: $\zeta=0$. Because

$$
\frac{\partial(f, \bar{f})}{\partial(\zeta, \bar{\zeta})}(0,0,0)=A B>0
$$

the implicit function theorem guarantees that this zero can be continued for nonzero but sufficiently small $\varepsilon$. That is to say, there exists a function $\varepsilon \zeta_{0}(\varepsilon)$ which is analytic at $\varepsilon=0$ such that

$$
f\left(\varepsilon \zeta_{0}(\varepsilon), \varepsilon \bar{\zeta}_{0}(\varepsilon), \varepsilon\right)=0 \text {. }
$$

Since $\varepsilon \zeta_{0}(\varepsilon)$ is a fixed point of the map $M$, it gives rise to a periodic orbit of the full Hamiltonian. Setting $\zeta=\hat{\zeta}+\varepsilon \zeta_{0}(\varepsilon)$ in formula (16) and dropping the carets again, we shift the fixed point into $\zeta=0$ without changing the general form of the map $M$. But the map $M$ has the form of a measure preserving perturbed twist. Moreover, this twist is non-degenerate in a sufficiently small annulus $0<\varrho<\varrho_{0}(\varepsilon)$ if $F^{\prime \prime}(0)=2 \kappa \neq 0$ and $\varepsilon$ is small enough. Here $\varrho_{0}$ can be assumed to be a continuous function of $\varepsilon$ for $\varepsilon>0$. Assume now that $V$ is an open neighborhood of the point $\varepsilon=\varepsilon_{0}>0, \varrho=0$ $\left(\varepsilon_{0}\right.$ small enough). Then $V$ contains a "cylinder" of the type

$$
\left\{\varrho, \varepsilon\left|\varrho \leqq \varrho_{1},\right| \varepsilon-\varepsilon_{0} \mid \leqq \delta ; \varrho_{1}<\min _{\left|\varepsilon_{0}-\varepsilon\right| \leqq \delta} \varrho_{0}(\varepsilon) ; \delta<\varepsilon_{0}\right\} .
$$

According to Moser's twist theorem, the annulus $0<\varrho<\varrho_{1}$ ( $\varepsilon$ fixed) contains an invariant curve : $C_{\varepsilon}$ surrounding the fixed point $\varrho=0$ of $M$. Because these curves can be assumed to depend continuously on $\varepsilon$ for $\left|\varepsilon-\varepsilon_{0}\right|<\delta$, the set

$$
\bigcup_{\left|\varepsilon-\varepsilon_{0}\right|<\delta} \text { Interior }\left(C_{\varepsilon}\right)
$$

is open. Moreover, it is contained in $V$ and invariant under $M$, proving the stability of the fixed point $\varepsilon=\varepsilon_{0}, \zeta=0$. The stability of the associated periodic orbit of the full Hamiltonian is proved.

\section{The Question of Stability of the Origin of $R^{4}$}

In contradistinction to the Hamiltonian studied in [1] our present Hamiltonian has a leading term that is indefinite. Thus, the stability of the origin of $R^{4}$ is no longer guaranteed and a separate investigation of its nature becomes necessary. In the case $n=2$ a criterion which allows a decision about the nature of this equilibrium point 
has been found by Sokol'skii [4]. Theorem 3 of the present section contains a generalization of his criterion to the case of general $n$. In other words, we shall assume that our Hamiltonian has the form

$$
K_{n}\left(M_{0}, M\right)=\sum_{j=p-1}^{n} M_{0}^{j} K_{n j}(M),
$$

where $p$ is an integer between 1 and $n$, defined by the condition that $K_{n p-1}$ is not identically zero [compare (1.25)]. In the symplectic chart $L, \alpha_{1}, R, \phi[\operatorname{see}(1.16)$ and (1.18)] our Hamiltonian takes the form

$$
H=P\left(\frac{L}{2}\right)+K_{n}\left(\frac{L}{2}, \sqrt{R(L+R)} \cos \phi, \sqrt{R(L+R)} \sin \phi, \frac{L}{2}+R\right)+\mathcal{O}_{2 n+1},
$$

where $\mathcal{O}_{2 n+1}$ represents a convergent power series in the variables $(L+R)^{1 / 2}, R^{1 / 2}$ that starts out with a term of order $2 n+1$ and whose coefficients are real analytic, periodic functions of $\alpha_{1}$ and $\phi$. Stretching the variables $z_{k}(k=1,2)$ by setting

$$
z_{k}=\varepsilon^{p} \hat{z}_{k} \quad(k=1,2),
$$

where $\varepsilon$ is a small positive number and $p$ is the integer defined in connection with formula (1), replaces the Hamiltonian $H$ by

$$
\hat{H}=\varepsilon^{-2 p} P\left(\varepsilon^{2 p} \frac{L}{2}\right)+\varepsilon^{2 p(n-1)} K_{n}+\mathcal{O}\left(\varepsilon^{p(2 n-1)}\right),
$$

where the arguments of $K_{n}$ are the same as in (2) except for the carets on $R$ and $L$. In the following we shall drop these carets again except for the one on $H$. Let $c$ be a real constant with the property $0<|c| \leqq 1$. On the energy surface

$$
\hat{H}=2 c \varepsilon, \text { i.e. } H=2 c \varepsilon^{2 p+1}
$$

$-L$ becomes a function of $\alpha_{1}, R, \phi, \varepsilon$ which is real analytic at $\varepsilon=0$, for all $\alpha_{1}, \phi$ and for $R$ restricted to some open interval with positive initial point. After dropping an irrelevant constant term we find that this function $\Lambda$ (not to be confused with the function denoted by the same symbol in Section 3) has a power series expansion in $\varepsilon$ that starts out as follows

$$
\Lambda\left(\alpha_{1}, R, \phi, \varepsilon\right)=\varepsilon^{q} c^{p-1} R^{r} Q(\phi)+\mathcal{O}\left(\varepsilon^{q+1}\right),
$$

where $q=(2 n-1) p-1, r=n-p+1$ and

$$
Q(\phi)=K_{n p-1}(\cos \phi, \sin \phi, 1) \text {. }
$$

The function $\Lambda$ together with the 2-form $d R \wedge d \phi$ determines the flow on the energy surface (3) with $\alpha_{1}$ serving as time parameter. The following is a generalization of a theorem of Sokol'skii:

Theorem 3. (i) If $Q(\phi)$ has a simple zero then the origin of $R^{4}$ is unstable.

(ii) If $Q(\phi)$ has no zero and if $p=1(2 \leqq p \leqq n-1)$ then the origin of $R^{4}$ is stable (weakly stable).

Remark. The term: "weakly stable" means that for almost all (in the sense of measure theory) initial conditions sufficiently close to the origin of $R^{4}$ the corresponding solution-curves do not leave a pre-assigned open neighborhood. 
Proof of (i). In order to prove (i) we assume first that $\phi_{0}$ is a simple zero of $Q(\phi)$ with $Q^{\prime}\left(\phi_{0}\right)<0$. We choose $\delta>0$ so small that $-Q_{0}=\sup _{\phi \in I_{\delta}} Q^{\prime}(\phi)<0$. Here, $I_{\delta}=\left[\phi_{0}-\delta, \phi_{0}+\delta\right]$. We will show that any solution starting in the interior of the domain

$$
\mathscr{D}_{c}=\left\{R, \phi \mid c \leqq R \leqq 2 c, \phi \in I_{\delta}\right\}
$$

on the energy surface (3) will eventually escape $\mathscr{D}_{c}$ through the outer circular part of its boundary. For this purpose we remark that up to a positive factor the vectorfield associated with the Hamiltonian (4) is

$$
r Q(\phi) \frac{1}{R} \frac{\partial}{\partial \phi}-Q^{\prime}(\phi) \frac{\partial}{\partial R}+\mathcal{O}(\varepsilon) .
$$

Because $-Q^{\prime}(\phi) \geqq Q_{0}>0$ and therefore $Q(\phi) \geqq Q_{0} \cdot\left(\phi_{0}-\phi\right)>0$ for $\phi_{0}-\delta \leqq \phi<\phi_{0}$ and $Q(\phi) \leqq-Q_{0} \cdot\left(\phi-\phi_{0}\right)<0$ for $\phi_{0}<\phi \leqq \phi_{0}+\delta$, we see that everywhere on the boundary of $\mathscr{D}_{c}$ except on the circular arc: $R=2 c, \phi \in I_{\delta}$ the vector-field points inside $\mathscr{D}_{c}$ as long as $\varepsilon$ is small enough. Because the radial component is positive everywhere in $\mathscr{D}_{c}$ as long as $\varepsilon$ is small enough it follows that every solution starting in the interior of $\mathscr{D}_{c}$ will eventually escape through the outer circular arc.

If $\phi_{0}$ is a simple zero of $Q(\phi)$ with $Q^{\prime}\left(\phi_{0}\right)>0$, then the time reversal (1.30) replaces $K_{n}\left(M_{0}, \boldsymbol{M}\right)$ by $\tilde{K}_{n}\left(M_{0}, M\right)=-K_{n}\left(-M_{0}, \boldsymbol{M}\right)$. The time reversed flow induced by $H$ on the energy surface

$$
\hat{H}=-2 c \varepsilon \quad(c>0)
$$

is again governed by a Hamiltonian of the type (4), where $Q(\phi)$ is replaced by $\tilde{Q}(\phi)=-Q(\phi)$. Hence, $\tilde{Q}^{\prime}\left(\phi_{0}\right)<0$ and we may proceed as before.

Proof of (ii). It is no retriction to assume that $Q(\phi)>0$ because according to what has been said above if $Q(\phi)<0$ a time reversal will replace $Q(\phi)$ by a trigonometric polynomial that is positive. With the help of the generating function

$$
S(\phi, J)=\frac{J}{J_{0}} \int_{0}^{\phi} \frac{d \phi}{[Q(\phi)]^{1 / r}}, J_{0}=\frac{1}{2 \pi} \int_{0}^{2 \pi} \frac{d \phi}{[Q(\phi)]^{1 / r}}
$$

we introduce action and angle variables $J$ and $\chi=\frac{\partial S}{\partial J}$. In these variables the Hamiltonian (4) takes the form

$$
\tilde{\Lambda}\left(\alpha_{1}, J, \chi, \varepsilon\right)=c^{(p-1)} \varepsilon^{q}\left(\frac{J}{J_{0}}\right)^{r}+\mathcal{O}\left(\varepsilon^{p+1}\right) .
$$

If $r \geqq 2$, i.e. $p \leqq n-1$ Moser's twist theorem [3] allows us to construct on each energy surface with $c \neq 0$ an invariant torus which prevents any solution that starts close to the origin of $R^{4}$ on this surface from escaping. For details of this construction in a similar situation see [5, pp. 43-45]. The possibility of this construction depends on the non-degeneracy of the twist which on the surface $H=0$ is only guaranteed if $p=1$. Thus, for $2 \leqq p \leqq n-1$ we cannot exclude the possibility of a solution starting on the surface $H=0$ from escaping a pre-assigned neighborhood of the origin of $R^{4}$. Because the set of points of such a surface has measure 0 our theorem is proved. 


\section{Classification of Periodic Orbits for $n=2$}

From now on we will assume that $n=2$. In Sections 5 and 6 we shall present a complete classification of all periodic orbits of our Hamiltonian that arise from c.p. of $K^{(2)}$ on $h^{2}$. It turns out that it is advantageous to distinguish two main cases: Cases I and II, depending on whether the matrix $\mathfrak{A}$ (see below) is diagonalizable with the help of a matrix $O \in \operatorname{SO}(2,1)$ or not. In Section 5 we will treat Case I, postponing Case II until Section 6.

A classification of periodic solutions is synonymous with a classification of critical points of $K^{(2)}$ on $h^{2}$. Right from the beginning we shall introduce a distinction between two classes of c.p. This distinction will make sense for general $n$ and was already considered in [1]. A c.p. will be called of second or first kind depending on whether the multiplier [defined in (2.4)] is an eigenvalue of $G \mathfrak{A}(\boldsymbol{e})$ or not.

If $n=2$ we can write $K^{(2)}(x)$ in the form

$$
K^{(2)}(\boldsymbol{x})=\frac{a_{0}}{2}+\langle\boldsymbol{x}, \boldsymbol{b}\rangle+\frac{1}{2}(\boldsymbol{x}, \mathfrak{A} \boldsymbol{x}),
$$

where $\boldsymbol{b}$ is a constant vector and $\mathfrak{A}$ a constant 3 by 3 matrix. The Equation (2.4) becomes

$$
(\lambda G-\mathfrak{A}) \boldsymbol{e}=G \boldsymbol{b},
$$

and the Hessian $\mathfrak{A}(\boldsymbol{e})$ equals $\mathfrak{O}$ independently of $\boldsymbol{e}$. By introducing the function

$$
\boldsymbol{e}(\lambda)=(\lambda-G \mathfrak{U})^{-1} \boldsymbol{b}
$$

which is defined on the resolvent set of the matrix $G \mathfrak{A}$ we see that a c.p. of the first kind is of the form $e\left(\lambda_{0}\right)$, where $\lambda_{0}$ is a simple [see (5) below] zero of the function

$$
g(\lambda)=1-\langle\boldsymbol{e}(\lambda), \boldsymbol{e}(\lambda)\rangle .
$$

An easy calculation using formula (2.10) with $\mathfrak{A}(e)$ replaced by $\mathfrak{A}$ yields the following expression for its index

$$
\text { ind } \boldsymbol{e}\left(\lambda_{0}\right)=\operatorname{sgn}\left[\operatorname{det}\left(\lambda_{0}-G \mathfrak{U}\right) g^{\prime}\left(\lambda_{0}\right)\right] \text {. }
$$

A glance at the Equation (2) shows that a c.p. of the second kind can only be present if the vector $\boldsymbol{b}$ is Lorentz-orthogonal (i.e. orthogonal with regard to the inner product $\langle\rangle$,$) to the eigenspace to the eigenvalue \lambda$ of the matrix $G \mathfrak{A}$. As already pointed out in Section $1, K^{(2)}$, as given in (1), is not uniquely determined by $H$. If we subject the $z_{k}$-variables $(k=1,2)$ to a transformation which replaces $Z$ by $U Z$, $U \in \mathrm{SU}(1,1)$ [see $(1.27)], \boldsymbol{x}$ is replaced by $O(U) \boldsymbol{x}, O(U) \in \mathrm{SO}^{\uparrow}(2,1)$ [see 1.28)] and consequently $\boldsymbol{b}$ by $O(U)^{-1} b$ and $\mathfrak{A}$ by $O(U)^{T} \mathfrak{Q} O(U)$. The question arises whether $\mathfrak{A}$ can be diagonalized by such a transformation. In order to answer this question we find it convenient to distinguish the following two possibilities.

(I) $G \mathfrak{U}$ has no eigenspace that coincides with an isotropic line.

(II) $G \mathfrak{A}$ has such an eigenspace.

Here, a line is called isotropic if it is spanned by an isotropic vector and a vector is called isotropic if $\langle\boldsymbol{x}, \boldsymbol{x}\rangle=0$. Borrowing from the vocabulary used in relativistic physics we also call such a vector null-like. 
Correspondingly, a vector $\boldsymbol{x}$ will be called time-like if $\langle\boldsymbol{x}, \boldsymbol{x}\rangle>0$ and space-like if $\langle\boldsymbol{x}, \boldsymbol{x}\rangle\left\langle 0\right.$. A Lorentz-basis of $R^{3}$ is a basis consisting of one time-like vector and two space-like vectors such that the inner product $\langle$,$\rangle in that basis is represented by$ the matrix $G$ [see (2.7)]. Before we formulate a necessary and sufficient condition under which we can diagonalize the matrix $\mathfrak{A}$ by means of a transformation $O(U)(U \in \mathrm{SU}(1,1))$ we want to emphasize that assumption I also excludes the existence of a complex isotropic eigenspace and therefore implies that all eigenvalues of the matrix $S=G \mathfrak{U}$ are real. Indeed, if the inner produce $\langle$,$\rangle is$ extended in the usual manner to a sesquilinear form over $\mathbb{C}^{3}$ (say, antilinear in the first and linear in the second argument) then $S$ is Hermitian with respect to this extended inner product. But this implies in the usual manner that all eigenvalues of $S$ to non-isotropic eigenvectors are necessarily real and that any complex eigenvector is necessarily isotropic (with respect to the properly extended inner product).

The following theorem addresses itself to the question raised above.

Theorem 4. (i) $\mathfrak{A}$ is diagonalized by a transformation $O(U)$ if and only if the assumption $I$ is satisfied. In that case there exists $U \in \mathrm{SU}(1,1)$ such that

$$
O^{T}(U) \mathfrak{H} O(U)=\operatorname{diag}\left(-a_{1},-a_{2}, a_{3}\right),
$$

where $a_{1}, a_{2}, a_{3}$ are the necessarily real eigenvalues of the matrix $S=G \mathfrak{U}$.

(ii) If the eigenvalues of $S$ are real and distinct the assumption I holds.

Proof of (i). Because the homomorphism $O: \mathrm{SU}(1,1) \rightarrow \mathrm{SO}^{\uparrow}(2,1)$ which associates $O(U)$ with $U$ is surjective, we only have to show that $\mathfrak{A}$ can be diagonalized by some $O \in \mathrm{SO}^{\uparrow}(2,1)$ if and only if $G \mathfrak{U}$ has no eigenspace that is spanned by an isotropic vector. Assume first $\mathfrak{A}$ is diagonalizable in this fashion, i.e. there exists $O \in \operatorname{SO}(2,1)$ such that $O^{T} \mathfrak{A} O=G D$, where $D$ is a diagonal matrix. Then, $O^{T} \mathfrak{A} O=O^{T} G O D$, i.e. $G \mathfrak{Q} O=O D$, i.e. the columns of $O$ form a basis of eigenvectors of $S$. Hence, $S$ has no eigenspace which is spanned by an isotropic vector. Assume now that $\mathfrak{A}$ satisfies condition $I$. $S$ has certainly an eigenvector $f$ which, according to assumption $I$, is or can be chosen to be non-isotropic. Its conjugate (i. e. Lorentz-orthogonal) plane $\mathscr{P}$ is also invariant under $S$ and does not contain $f$. If $f$ is time-like, $\mathscr{P}$ is space-like and the restriction of $S$ to $\mathscr{P}$ is symmetric with respect to the negative definite inner product obtained by restricting $\langle$,$\rangle to \mathscr{P}$ and therefore possesses two orthogonal vectors : $f_{1}$, $\boldsymbol{f}_{2}$ with the property $\left\langle\boldsymbol{f}_{i}, \boldsymbol{f}_{i}\right\rangle=-1(i=1,2)$. Hence, $\boldsymbol{f}_{1}, \boldsymbol{f}_{2}, \boldsymbol{f}_{3}= \pm \boldsymbol{f}$ is a Lorentz-basis. If we choose the sign in front of $f$ such that the $z$-component of $f_{3}$ is positive, then the matrix $O$ whose columns are the vectors $f_{1}, f_{2}, f_{3}$ belongs to $\mathrm{SO}^{\dagger}(2,1)$ and has the property $S O=O D, D=\operatorname{diag}\left(a_{1}, a_{2}, a_{3}\right)$, where $a_{i}$ is the eigenvalue of $S$ to the eigenvector $f_{i}$. However, the last relation is easily seen to be equivalent to (6): $O^{T} \mathfrak{A} O=O^{T} G O D=G D$. Thus, the statement (i) is proved provided $f$ is time-like. However, if $f$ is space-like its conjugate plane $\mathscr{P}$ cuts the cone of isotropic vectors. Because $S$ leaves this plane invariant its restriction to $\mathscr{P}$ has another eigenvector which, again by assumption $I$, is or can be chosen to be non-isotropic. If this eigenvector is time-like it can take the plane of $f$ in the first considered case. If it is space-like its conjugate vector is a time-like eigenvector of $S$ which therefore can take over the role of $f$ in the first considered case. Statement (i) of Theorem (4) is proved. 
As to the Statement (ii) we simply remark that under the assumption that the $a_{i}$ 's are mutually distinct the eigenvectors of $S$ must be linearly independent. Because they are also mutually Lorentz-orthogonal it follows that the condition $I$ is satisfied. Q.E.D.

In the remaining part of Section 5 we will assume that $\mathscr{U}$ satisfies condition $I$ and thus can be assumed to be in diagonal form. The functions defined in (1) and (3)-(5) [see also (2.10)] assume the following simple forms:

$$
\begin{aligned}
K^{(2)}(\boldsymbol{x}) & =\frac{a_{0}}{2}+\langle\boldsymbol{x}, \boldsymbol{b}\rangle+\frac{1}{2}\left(-a_{1} x^{2}-a_{2} y^{2}+a_{3} z^{2}\right), \\
\boldsymbol{e}(\lambda) & =\left(\frac{b_{1}}{\lambda-a_{1}}, \frac{b_{2}}{\lambda-a_{2}}, \frac{b_{3}}{\lambda-a_{3}}\right), \\
g(\lambda) & =1+\frac{b_{1}^{2}}{\left(\lambda-a_{1}\right)^{2}}+\frac{b_{2}^{2}}{\left(\lambda-a_{2}\right)^{2}}-\frac{b_{3}^{2}}{\left(\lambda-a_{3}\right)^{2}}, \\
\Delta(\boldsymbol{e}) & =\frac{1}{2}\left(\lambda-a_{1}\right)\left(\lambda-a_{2}\right)\left(\lambda-a_{3}\right) g^{\prime}(\lambda) \\
& =e_{3}^{2}\left(\lambda-a_{1}\right)\left(\lambda-a_{2}\right)-e_{1}^{2}\left(\lambda-a_{2}\right)\left(\lambda-a_{3}\right)-e_{2}^{2}\left(\lambda-a_{1}\right)\left(\lambda-a_{3}\right),
\end{aligned}
$$

where $\boldsymbol{e}=\left(e_{1}, e_{2}, e_{3}\right)$.

Let us first assume that the $a_{i}$ 's are mutually distinct. This case will be referred to as Case $\mathrm{I}_{0}$, whereas the cases in which some of the $a_{i}$ 's coincide will be collected under the heading Case $\mathrm{I}_{1}$.

In the Case $\mathrm{I}_{0}$, to which we now turn, the classification of the c.p. of $K^{(2)}$ on $h^{2}$ is facilitated by the introduction of the following quantities:

$$
\begin{aligned}
& \Delta_{1}=-\left(a_{1}-a_{2}\right)\left(a_{1}-a_{3}\right) \\
& \Delta_{2}=-\left(a_{2}-a_{1}\right)\left(a_{2}-a_{3}\right) \\
& \Delta_{3}=\left(a_{3}-a_{1}\right)\left(a_{3}-a_{2}\right),
\end{aligned}
$$

and

$$
\varepsilon_{i}=\operatorname{sgn} \Delta_{i} \quad(i=1,2,3) .
$$

For calculation with the $\varepsilon_{i}$ 's $(i=1,2,3)$ we have to observe the following basic rules :

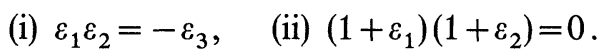

They imply the additional rule

(iii) $1+\varepsilon_{1}+\varepsilon_{2}=\varepsilon_{3}$

which will be particularly useful in the following.

We also distinguish the two cases in which $b_{3} \neq 0$ and $b_{3}=0$ and note first the following facts:

If $b_{3}=0$ then there exists precisely one pair of c.p. of $K^{(2)}$ on $h^{2}$, namely

$$
f_{3}^{ \pm}=\left(\frac{b_{1}}{a_{3}-a_{1}}, \frac{b_{2}}{a_{3}-a_{2}}, \pm\left[g\left(a_{3}\right)\right]^{1 / 2}\right) \text {. }
$$

It is a pair of c.p. of the second kind with multiplier $\lambda=a_{3}$, and with both members of the pair having index $\varepsilon_{3}$. 
If $b_{3} \neq 0$ and $g\left(a_{i}\right)<0(i=1,2)$ the following pair of c.p. of the second kind is present

$$
\begin{aligned}
& \boldsymbol{f}_{1}^{ \pm}=\left( \pm\left[-g\left(a_{1}\right)\right]^{1 / 2}, \frac{b_{2}}{a_{1}-a_{2}}, \frac{b_{3}}{a_{1}-a_{3}}\right) \text { if } i=1 \\
& \boldsymbol{f}_{2}^{ \pm}=\left(\frac{b_{1}}{a_{2}-a_{1}}, \pm\left[-g\left(a_{2}\right)\right]^{1 / 2}, \frac{b_{3}}{a_{2}-a_{3}}\right) \text { if } i=2,
\end{aligned}
$$

with index ind $f_{i}^{ \pm}=\varepsilon_{i}(\mathrm{i}=1,2)$.

If $b_{3} \neq 0$ there always exist c.p. of the first kind corresponding to zeros of the function $g$. Indeed, if $b_{3} \neq 0$ this function possesses always at least two zeros: $\lambda_{1}$ being located on the real axis on the same side relative to $a_{3}$ as $a_{1}$ and $\lambda_{0}$ being located on the opposite side.

The situation with regard to c.p. of $K^{(2)}$ on $h^{2}$ in the Case $\mathrm{I}_{0}, b_{3} \neq 0$ is summarized in Table 1 which classifies the c.p. $\boldsymbol{e}\left(\lambda_{0}\right), \boldsymbol{e}\left(\lambda_{1}\right)$ of the first kind and the c.p. $f_{i}^{ \pm}(i=1,2)$ of the second kind according to the signs of $g\left(a_{1}\right)$ and $g\left(a_{2}\right)$. In reading the table the following conventions have to be observed: 1) $\operatorname{sgn} g\left(a_{i}\right)=+1$ if $g\left(a_{i}\right) \geqq 0$ or if $\lim _{\lambda \rightarrow a_{i}} g(\lambda)=\infty$. 2) The appearance of a symbol * in a column means that the c.p. that heads the column is not present. All other symbols mean that the c.p. is present with the indicated index. 3) The third column gives a condition on the vector $b$ which has to be satisfied in order that the given sign combination of the $g\left(a_{i}\right)$ 's $(i=1,2)$ is possible. 4) The question of the presence of additional c.p. of the first kind is relegated to comments following Table 1.

Table 1

\begin{tabular}{lllcccc}
\hline $\operatorname{sgn} g\left(a_{1}\right)$ & sgn $g\left(a_{2}\right)$ & $\begin{array}{l}\text { Condition } \\
\text { on vector } \boldsymbol{b}\end{array}$ & $\boldsymbol{e}\left(\lambda_{0}\right)$, & $\boldsymbol{e}\left(\lambda_{1}\right)$, & $\boldsymbol{f}_{1}^{ \pm}$, & $\boldsymbol{f}_{2}^{ \pm}$ \\
\hline+ & + & None & $\varepsilon_{3}$ & $\varepsilon_{3}$ & $*$ & $*$ \\
+ & - & $b_{2}=0$ & 1 & $\varepsilon_{1}$ & $*$ & $\varepsilon_{2}$ \\
- & + & $b_{1}=0$ & $\varepsilon_{3}$ & $-\varepsilon_{1}$ & $\varepsilon_{1}$ & $*$ \\
- & - & $b_{1}=b_{2}=0$ & 1 & 1 & $\varepsilon_{1}$ & $\varepsilon_{2}$ \\
\hline
\end{tabular}

Comment on Row 1. One or two (+ - )-pairs (elliptic-hyperbolic pairs) of c.p. of the first kind may be present in addition to $e\left(\lambda_{0}\right)$ and $e\left(\lambda_{1}\right)$.

Comment on Row 2. If $\varepsilon_{1}=-1$ one additional (+-)-pair may be present. If $\varepsilon_{1}=1$ one additional $(++)$-pair must be present.

Comment on Row 3. If $\varepsilon_{2}=-1$ one additional (+-)-pair may be present. If $\varepsilon_{2}=1$ one additional $(++)$-pair must be present.

Comment on Row 4. In this case the following explicit formulae hold:

$$
\begin{aligned}
& \lambda_{1}=a_{3}+\operatorname{sgn}\left(a_{1}-a_{3}\right)\left|b_{3}\right|, \\
& \lambda_{0}=a_{3}-\operatorname{sgn}\left(a_{1}-a_{3}\right)\left|b_{3}\right|, \\
& \boldsymbol{e}\left(\lambda_{1}\right)=\operatorname{sgn}\left[b_{3}\left(a_{1}-a_{3}\right)\right] \boldsymbol{k} ; \quad \boldsymbol{e}\left(\lambda_{0}\right)=-\operatorname{sgn}\left[b_{3}\left(a_{1}-a_{3}\right)\right] \boldsymbol{k} .
\end{aligned}
$$


Some general observations in the Case $\mathrm{I}_{0}$ are expressed in the following theorem.

Theorem 5. If all eigenvalues of the matrix $G \mathfrak{U}$ are real and distinct $\left(\mathrm{Case}_{0}\right)$, then

(i) the total index of all c.p. of $K^{(2)}$ on $h^{2}$ is always $2 \varepsilon_{3}$.

(ii) on each energy surface (for sufficiently small absolute value of the energy) there exists at least one periodic orbit.

Proof. We prove statement (i) for the second row of Table 1 leaving its proof for the other three rows to the reader.

By taking into account the comment on row 2 made above we find that the total index of Row 2 is : $3+\varepsilon_{1}+2 \varepsilon_{2}=2\left(1+\varepsilon_{1}+\varepsilon_{2}\right)=2 \varepsilon_{3}$ if $\varepsilon_{1}=1$ and $1+\varepsilon_{1}+2 \varepsilon_{2}=2 \varepsilon_{2}$ $=2 \varepsilon_{3}$ if $\varepsilon_{1}=-1$. Here, we made use of the rules (iii) and.(i) of our $\varepsilon$-calculus.

In order to prove statement (ii) of Theorem 5 we have only to show that at least one of all the c.p. that the function $K^{(2)}$ possesses on $h^{2}$ lies on $h_{+}^{2}$ and at least one lies on $h_{-}^{2}$. An inspection of Equation (13) reveals that this is certainly the case if $b_{3}=0$. However, as observed earlier (see Table 1), if $b_{3} \neq 0$ there exist at least two c.p. of the first kind $e\left(\lambda_{0}\right)$ and $e\left(\lambda_{1}\right)$, and because, by definition, $\lambda_{0}$ and $\lambda_{1}$ are numbers that on the real axis are located on different sides of $a_{3}$, Equation (8) shows that $e\left(\lambda_{0}\right)$ and $e\left(\lambda_{1}\right)$ lie on different sheets of the hyperboloid $h^{2}$. The theorem is proved.

At this point the question arises whether in the Case $\mathrm{I}_{0}$ the origin of $R^{4}$ is a stable or an unstable equilibrium point. The following theorem reveals an interesting connection between the answer to this question and the total index of all c.p.

Theorem 6. If all eigenvalues of the matrix $G \mathfrak{U}$ are real and distinct $\left(\mathrm{Case}_{0}\right)$, then the origin of $R^{4}$ is stable/unstable if and only if the total index of all c.p. of $K^{(2)}$ on $h^{2}$ is $2 /-2$, that is to say, if $\varepsilon_{3}=1 /-1$.

Proof. An easy calculation using (7) shows that in the Case $\mathrm{I}_{0}$ the Sokol'skii-function [defined in (4.5)] is $Q(\phi)=\alpha+\beta \cos 2 \phi$, where $\alpha=a_{3}-\frac{1}{2}\left(a_{1}+a_{2}\right), \beta=\frac{1}{2}\left(a_{2}-a_{1}\right)$. Theorem 6 is an immediate consequence of Theorem 3 because $Q(\phi)$ either satisfies the condition (i) or the condition (ii) of that theorem depending on whether $|\beta|>|\alpha|$, i.e. $\varepsilon_{3}=-1$ or $|\beta|<|\alpha|$, i.e. $\varepsilon_{3}=+1 .(|b|=|a|$ is excluded by the assumption that the $a_{i}$ 's are all distinct.) Q.E.D.

Before concluding this section, we turn to a short discussion of the Case $I_{1}$ in which some of the $a_{i}$ 's coincide.

If $a_{1}=a_{2}=a_{3} \equiv a$, then by a transformation of type (1.26) we can achieve $a=0$ and by a transformation of type (1.27) the vector $\boldsymbol{b}$ (which we assume to be different from zero) can be brought into one of the three forms $(0,0, b),(b, 0,0)$ or $(b, 0, b)$ depending on whether $b$ is time-, space-, or null-like. There are no c.p. except in the first case in which the pair of elliptic c.p. $\pm \boldsymbol{k}$ is present. However, the stability of the corresponding family of periodic orbits is not guaranteed because the expression (3.9) vanishes. Also, we cannot assert the stability of the origin of $R^{4}$ because $p=n=2$.

In the second case ( $b=$ space-like) this equilibrium point is unstable as follows from statement (i) of Theorem 3 and the fact that the Sokol'skii-function is $Q(\phi)$ $=-b \cos \phi$. (See also [7].) 
In the following we will assume $a_{1} \neq a_{3}$. If not all $a_{i}$ 's coincide this can always be achieved by a transformation of type (1.27) inducing a rotation about the $z$-axis. Under this assumption and if $b_{3} \neq 0$ the zeros $\lambda_{0}, \lambda_{1}$ of the function $g$ which were introduced earlier continue to be well defined.

Next, assume $a_{1}=a_{2} \equiv a \neq a_{3}$, hence $\varepsilon_{3}=1$. If $b_{3}=0$ the only two c.p. are the two elliptic c.p. $f_{3}^{ \pm}$given in (13).

If $b_{3} \neq 0$ the first or last row of Table 1 applies (with $\varepsilon_{3}=1$ ) depending on whether $g(a) \geqq 0$ or $g(a)<0$ with the exception that in the latter case the four c.p. $f_{i}^{ \pm}(i=1,2)$ are replaced by the one-parameter family of degenerate c.p.

$$
f_{12}(\chi)=\left([-g(a)]^{1 / 2} \cos \chi,[-g(a)]^{1 / 2} \sin \chi, \frac{b_{3}}{a-a_{3}}\right) \quad(0 \leqq \chi<2 \pi) .
$$

Theorem 6 (and its proof) remains valid in this case (with $\varepsilon_{3}=1$ ) and it implies that the origin of $R^{4}$ is stable.

Finally, assume $a_{2}=a_{3} \equiv a \neq a_{1}$. If $\left|b_{2}\right|>\left|b_{3}\right|$ no c.p. exist. If $\left|b_{2}\right|=\left|b_{3}\right|$ only the one-parametric family of degenerate c.p. of the second kind

$$
f_{23}(\chi)=\left(\frac{b_{1}}{a_{3}-a_{1}},[g(a)]^{1 / 2} \operatorname{sh} \chi,[g(a)]^{1 / 2} \operatorname{ch} \chi\right)
$$

$(\chi \in R)$ is present. On the other hand, if $\left|b_{3}\right|>\left|b_{2}\right|$ we have $\operatorname{sgn} g\left(a_{2}\right)<0$ (actually: $\lim _{\lambda \rightarrow a_{2}} g(\lambda)=-\infty$ ) and $\varepsilon_{1}=-1$, and the second or fourth row of Table 1 applies (with $\left.\varepsilon_{1}=-1\right)$ depending on whether $g\left(a_{1}\right) \geqq 0$ or $g\left(a_{1}\right)<0$, except that we have to ignore the column headed by $\boldsymbol{f}_{2}^{ \pm}$.

The total index is zero and the stability of the origin of $R^{4}$ depends on higher order terms in the expansion of our Hamiltonian $H$.

\section{Classification of Periodic Orbits for $n=2$}

We continue with the classification of c.p. that we began in Section 5 with a treatment of the Case II. In other words, we work under the assumption that the matrix $S=G \mathfrak{U}$ has an isotropic eigenspace. First we shall assume that this eigenspace is real $\left(\right.$ Case $\mathrm{II}^{\mathrm{R}}$ ) postponing the case of a complex (isotropic) eigenspace $\left(\right.$ Case $\mathrm{II}^{\mathrm{C}}$ ) to the end of the present section.

In the Case $\mathrm{II}^{\mathrm{R}}$ to which we now turn we may assume that the isotropic eigenspace is spanned by a vector $f_{3}$ whose third component is positive. Let $a$ be the corresponding eigenvalue. The plane $\mathscr{P}$ conjugate to $f_{3}$ which is invariant under $S$ coincides with the tangent plane to the cone of isotropic vectors along $f_{3}$. We distinguish two cases

Case $\mathrm{II}_{0}^{R}$. $\mathscr{P}$ contains an eigenvector $f_{1}$ of $S$ linearly independent of $f_{3}$. The corresponding eigenvalue will be denoted by $a_{1}$.

Case $\mathrm{II}_{1}^{R}$. No such eigenvector exists. This case can only be present if the restriction of $S$ to $\mathscr{P}$ has a degenerate eigenvalue, i.e. $a_{1}=a$.

The following theorem gives the normal forms of the matrix $\mathfrak{A}$ on which our classification of c.p. in the Case $\mathrm{II}^{\mathrm{R}}$ will be based. 
Theorem 7. If $\mathfrak{A}$ satisfies condition $\mathrm{II}^{\mathrm{R}}$, then there exists a transformation $T$ such that

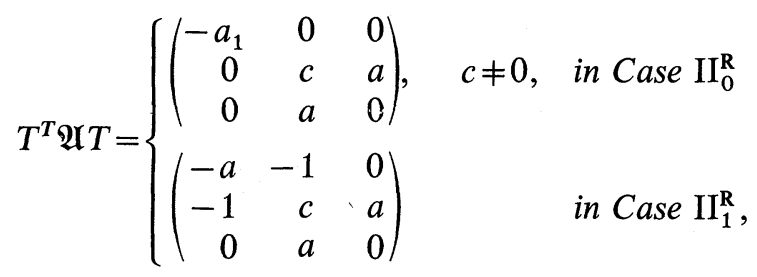

and

$$
T^{T} G T=\hat{G} \equiv\left(\begin{array}{rrr}
-1 & 0 & 0 \\
0 & 0 & 1 \\
0 & 1 & 0
\end{array}\right) .
$$

Remark. Obviously, $T$ cannot be induced by a transformation $U \in \mathrm{SU}(1,1)$ of the $z_{k}$-variables $(k=1,2)$. However, if we multiply $T$ with a matrix

$$
W= \pm\left(\begin{array}{ccc}
1 & 0 & 0 \\
0 & 2^{-1 / 2} & 2^{-1 / 2} \\
0 & -2^{-1 / 2} & 2^{-1 / 2}
\end{array}\right)
$$

then, by an appropriate choice of the sign in the last equation, we can arrange that $O=T W \in \mathrm{SO}^{\uparrow}(2,1)$ and therefore $O$ can be induced by a canonical transformation of the $z_{k}$-variables $(k=1,2)$. Thus, the normal form of $\mathfrak{A}$ that can be obtained by such a canonical transformation is

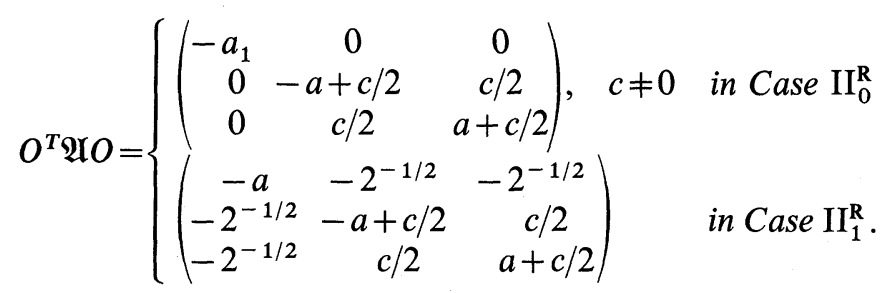

Proof of Theorem 7, Case $\mathrm{II}_{0}^{\mathrm{R}}$. It is clear from the definition of $f_{1}$ that we can assume $\left\langle f_{1}, f_{3}\right\rangle=0,\left\langle f_{1}, f_{1}\right\rangle=-1$. The plane conjugate to $f_{1}$ cuts the cone of isotropic vectors along $f_{3}$ and another null-like vector $f_{2}$ which we normalize such that $\left\langle\boldsymbol{f}_{2}, \boldsymbol{f}_{3}\right\rangle=1$. Clearly, the inner product $\langle$,$\rangle is represented in the basis \boldsymbol{f}_{1}, \boldsymbol{f}_{2}, \boldsymbol{f}_{3}$ by the matrix $\hat{G}$ (defined in Theorem 7 ) and if $T$ is the matrix with this basis as its columnvectors, we have

$$
S T=T N, \text { where } N=\left(\begin{array}{ccc}
a_{1} & 0 & 0 \\
0 & a_{2} & 0 \\
0 & c & a
\end{array}\right)
$$

and hence, $T^{T} \mathfrak{U} T=\hat{G} N$.

The symmetry of $\hat{G} N$ implies $a_{2}=a$, and $c \neq 0$ follows from our assumption II. By the replacement $|c|^{1 / 2} f_{3} \rightarrow f_{3},|c|^{-1 / 2} f_{2} \rightarrow f_{2}$ we can even normalize $c$ to become \pm 1 . 
Case $\mathrm{II}_{1}^{\mathrm{R}}$. We proceed precisely as in the previous case except that we can no longer assume that the vector $f_{1}$ with the properties $\left\langle f_{1} f_{3}\right\rangle=0,\left\langle f_{1}, f_{1}\right\rangle=-1$ is an eigenvector of $S$ and accordingly that the vector

$$
S f_{2}=d f_{1}+a_{2} f_{2}+c f_{3}
$$

may have all three components different from zero. Proceeding as in the C'ase $\mathrm{II}_{0}^{\mathrm{R}}$ we find

$$
T^{T} \mathfrak{U} T=\left(\begin{array}{rrr}
-a & -d & 0 \\
-d & c & a \\
0 & a & 0
\end{array}\right),
$$

where $d \neq 0$ follows from our assumption $\mathrm{II}_{1}^{\mathrm{R}}$. If we also take into account that the replacement $d \boldsymbol{f}_{3} \rightarrow \boldsymbol{f}_{3}, d^{-1} \boldsymbol{f}_{2} \rightarrow \boldsymbol{f}_{2}$ replaces $d$ by 1 (with a simultaneous renormalization of $c$ ) our proof of Theorem 7 is complete.

At this stage we are ready for the classification of the c.p. of $K^{(2)}$ on $h^{2}$ under the assumption $\mathrm{II}^{\mathrm{R}}$. Thus, we will assume that the matrix entering into the expression (5.1) is in one of the normal forms (1) and that the inner product $\langle$,$\rangle is represented$ by the matrix $\hat{G}$. We first treat the Case $\mathrm{II}_{0}^{\mathrm{R}}$. We assume that $c$ is normalized to \pm 1 . The Equation (5.2) become

$$
\left(\lambda-a_{1}\right) e_{1}=b_{1}, \quad(\lambda-a) e_{2}=b_{2}, \quad(\lambda-a) e_{3}=b_{3}+c e_{2},
$$

where $e_{1}, e_{2}, e_{3}$ are the components of the vector $\boldsymbol{e}$. The functions defined in (5.3), (5.4), and (2.10) take the form

$$
\begin{aligned}
& e(\lambda)=\left(\frac{b_{1}}{\lambda-a_{1}}, \frac{b_{2}}{\lambda-a}, \frac{b_{3}}{\lambda-a}+\frac{c b_{2}}{(\lambda-a)^{2}}\right), \\
& g(\lambda)=1+\frac{b_{1}^{2}}{\left(\lambda-a_{1}\right)^{2}}-\frac{2 c b_{2}^{2}}{(\lambda-a)^{3}}-\frac{2 b_{2} b_{3}}{(\lambda-a)^{2}}, \\
& \Delta(e)=-e_{1}^{2}(\lambda-a)^{2}+2 e_{2} e_{3}(\lambda-a)\left(\lambda-a_{1}\right)+c\left(\lambda-a_{1}\right) e_{2}^{2} .
\end{aligned}
$$

For the index of a c.p. of the first kind we use Formula (5.5) which in the present case simplifies to:

$$
\text { ind } e\left(\lambda_{0}\right)=\operatorname{sgn}\left[\left(\lambda_{0}-a\right) g^{\prime}\left(\lambda_{0}\right)\right] \text {. }
$$

First we treat the subcase $a_{1} \neq a$ which we call the Case $\mathrm{II}_{00}^{\mathrm{R}}$. We set

$$
\gamma=\operatorname{sgn}\left[c\left(a-a_{1}\right)\right]
$$

If $b_{2}=0$, then $g(\lambda)>0$ and no c.p. of the first kind exists. If also $b_{3}=0$ then there is no c.p. at all. However, if $b_{2}=0$ and $b_{3} \neq 0$ then there exists precisely one c.p. of the second kind

$$
f_{3}=\left(\frac{b_{1}}{a-a_{1}},-\frac{b_{3}}{c}, \frac{-c}{2 b_{3}} g(a)\right)
$$


with index $\gamma$. During the remainder of the discussion of the Case $\mathrm{II}_{00}^{\mathrm{R}}$ we shall assume $b_{2} \neq 0$. If also $b_{1}=0$ and $g\left(a_{1}\right)<0$ the hyperbolic pair of c.p. of the second kind

$$
f_{1}^{ \pm}=\left(\left[-g\left(a_{1}\right)\right]^{1 / 2}, \frac{b_{2}}{a_{1}-a}, \frac{b_{3}}{a_{1}-a}+\frac{c b_{2}}{\left(a_{1}-a\right)^{2}}\right)
$$

makes its appearance.

In the following let $I_{\infty}(a)$ be the semi-infinite open interval with (finite) endpoint a such that $a_{1} \in I_{\infty}(a)$ and let $I\left(a, a_{1}\right)$ be the closed interval with endpoints $a, a_{1}$. The function $g$ has always at least one zero $\lambda_{0}$ and we have $\lambda_{0} \in \mathrm{I}_{\infty}(\mathrm{a})$ or $\lambda_{0} \notin I_{\infty}(a)$ depending on whether $\gamma=-1$ or $\gamma=1$. The situation with regard to c.p. of $K^{(2)}$ on $h^{2}$ in the Case $\mathrm{II}_{00}^{\mathrm{R}}, b_{2} \neq 0$ is summarized in Table 2 and the comments following it. (The table has to be read in the same way as Table 1.).

Table 2

\begin{tabular}{lllrr}
\hline$\gamma$ & sgn $g\left(a_{1}\right)$ & $\begin{array}{l}\text { Condition } \\
\text { on vector } \boldsymbol{b}\end{array}$ & $\boldsymbol{e}\left(\lambda_{0}\right)$ & $\boldsymbol{f}_{1}^{ \pm}$ \\
\hline+ & + & None & 1 & $*$ \\
+ & - & $b_{1}=0$ and $(12)$ & 1 & -1 \\
- & + & None & -1 & $*$ \\
- & - & $b_{1}=0$ & 1 & -1 \\
\hline
\end{tabular}

Comments with regard to additional c.p. of the first kind:

On Row 1. There may exist one or two additional (+-)-pairs of the first kind corresponding to zeros of $g$ in $I_{\infty}(a)$.

On Row 2. There exists always one additional pair of elliptic c.p. of the first kind corresponding to two zeros of $g$ in $I_{\infty}(a)$ with $a_{1}$ lying in between them. Besides having vanishing first component the vector $\boldsymbol{b}$ must satisfy

$$
\langle b, b\rangle^{3}>27 b_{2}^{4} \text {. }
$$

On Row 3. Same comment as on row 1 except that possible zeros of $g$ other than $\lambda_{0}$ are located outside $I\left(a, a_{1}\right)$.

On Row 4. An additional (+ -)-pair of c.p. of the first kind corresponding to zeros of $g$ outside $I\left(a, a_{1}\right)$ exists precisely if the vector $\boldsymbol{b}$ satisfies (12).

In deriving the results contained in these comments the following formula proves to be useful: If $g(\lambda)=0$ then

$$
g^{\prime}(\lambda)=\frac{2}{\lambda-a}\left[1+e_{1}^{2} \frac{a-a_{1}}{\lambda-a_{1}}+e_{2}^{2} \frac{c}{\lambda-a}\right] .
$$

For the derivation of condition (12) see also the treatment of Case $\mathrm{II}_{01}^{\mathrm{R}}$ below. 
Theorem 8. In the Case $\mathrm{II}_{00}^{\mathrm{R}}$ the following statements hold:

(i) The total index of all c.p. is always $\gamma$.

(ii) If $\gamma=-1$ the origin of $R^{4}$ is unstable.

Proof. The first statement follows from an inspection of Table 2 taking into account the subsequent comments.

A simple calculation using the normal form (2) of our matrix $\mathfrak{A}$ yields the following Sokol'skii-function in the Case $\mathrm{II}_{00}^{\mathrm{R}}$ :

$$
Q(\phi)=4 \sin ^{2} \frac{\psi}{2}\left[\left(a-a_{1}\right) \cos ^{2} \frac{\psi}{2}+\frac{\mathrm{c}}{2} \sin ^{2} \frac{\psi}{2}\right]
$$

with $\psi=\phi-\frac{3 \pi}{2}$. Statement (ii) of Theorem 8 is an immediate consequence of statement (i) of Theorem 3. Notice that in the case $\gamma=1$ the nature of the origin of $R^{4}$ depends on higher order terms in the expansion of the Hamiltonian $H$.

Now, let us turn to the Case $\mathrm{II}_{01}^{\mathrm{R}}$, i.e. to that subcase of Case $\mathrm{II}_{0}^{\mathrm{R}}$ in which $a_{1}=a$. We set $\mu=c(\lambda-a)^{-1}$ and find from (5) and (6)

$$
\begin{aligned}
& \boldsymbol{e}(\lambda) \equiv \tilde{\boldsymbol{e}}(\mu)=\mu \cdot c\left(b_{1}, b_{2}, b_{3}+b_{2} \mu\right) \\
& g(\lambda) \equiv \tilde{g}(\mu)=1-\langle\boldsymbol{b}, \boldsymbol{b}\rangle \mu^{2}-2 b_{2}^{2} \mu^{3} .
\end{aligned}
$$

Thus, a c.p. of the first kind is given by $\tilde{\boldsymbol{e}}\left(\mu_{0}\right)$, where $\mu_{0}$ is a simple zero of $\tilde{g}$. Its index is

$$
\text { ind } \tilde{\boldsymbol{e}}\left(\mu_{0}\right)=-\operatorname{sgn}\left(\mu_{0} \tilde{g}^{\prime}\left(\mu_{0}\right)\right) \text {, }
$$

as follows from (8).

If $b_{2} \neq 0$, a study of the polynomial $\tilde{g}$ shows that there exists an elliptic c.p. of the first kind is joined by a (+-)-pair of c.p. of the first kind precisely if the vector $\boldsymbol{b}$ satisfies the inequality (12).

If $b_{2}=0$ there are no c.p. of the first kind. Actually, there are no c.p. at all except for the degenerate c.p.

$$
f_{3}=\left(0,-\frac{b_{3}}{c}, \frac{-c}{2 b_{3}}\right)
$$

which is present provided that $b_{1}=0, b_{3} \neq 0$.

The stability of the origin of $R^{4}$ depends on higher order terms in the expansion of the Hamiltonian $H$.

Next, we discuss the Case $\mathrm{II}_{1}^{\mathrm{R}}$. We set $\mu=(\lambda-a)^{-1}$ and obtain

$$
\tilde{\boldsymbol{e}}(\mu)=\mu\left(b_{1}+b_{2} \mu, b_{2}, b_{3}+c b_{2} \mu-b_{1} \mu-b_{2} \mu^{2}\right)
$$

and

$$
\tilde{g}(\mu)=1+3 b_{2}^{2} \mu^{4}+2 b_{2} \beta \mu^{3}-\langle b, b\rangle \mu^{2},
$$

where

$$
\beta=2 b_{1}-c b_{2} .
$$


If $b_{2}=0$, no c.p. exist, unless also $b_{1} \neq 0$, in which case the hyperbolic c.p.

$$
f_{3}=\left(b_{3}-c b_{1},-b_{1},-\frac{1+\left(b_{3}-c b_{1}\right)^{2}}{2 b_{1}}\right)
$$

is present. In the remainder of the discussion of the present case, we shall assume $b_{2} \neq 0$. We introduce the two numbers

$$
\mu_{ \pm}=\left(4 b_{2}\right)^{-1}\left[-\beta \pm\left(\beta^{2}+\frac{8}{3}\langle\boldsymbol{b}, \boldsymbol{b}\rangle\right)^{1 / 2}\right] \text {. }
$$

If $\boldsymbol{b}$ is time-like the function $\tilde{g}$ has a local minimum at each of the positions $\mu=\mu_{ \pm}$ and it follows that the number of $(+-)$-pairs of c.p. of the first kind agrees with the number of minus signs in the pair of numbers: $\operatorname{sgn} g\left(\mu_{-}\right), \operatorname{sgn} g\left(\mu_{+}\right)$. On the other hand, if the number $\langle\boldsymbol{b}, \boldsymbol{b}\rangle$ lies in the half-open interval $\left(-\frac{3}{8} \beta^{2}, 0\right]$ and if $\beta \neq 0$ then $\tilde{g}$ has a local minimum only at one of the two positions $\mu_{ \pm}$, namely at $\mu_{+}$if $\beta<0$ and at $\mu_{-}$if $\beta>0$. Thus, one $(+-)$-pair of c.p. is present precisely if this minimum is negative. If finally $\langle\boldsymbol{b}, \boldsymbol{b}\rangle \leqq-\frac{3}{8} \mu^{2}$ no c.p. exists.

Again, the Sokol'skii-function corresponding to this case is calculated using the normal form (2) of the matrix $\mathfrak{A}$. We obtain

$$
Q(\phi)=2 \sin ^{3} \frac{\psi}{2}\left(c \sin \frac{\psi}{2}-2^{3 / 2} \cos \frac{\psi}{2}\right),
$$

where $\psi=\phi-\frac{3 \pi}{2}$. Because it possesses always a simple zero, Theorem 3 implies that in the Case $\mathrm{II}_{1}$ the origin of $R^{4}$ is always unstable.

Finally, we turn to the Case $\mathrm{II}^{\mathrm{C}}$. In this case $S=G \mathfrak{U}$ has a complex eigenvalue $c+i a(a>0)$ and the corresponding eigenvector

$$
f=f_{2}+i f_{3}\left(f_{3} \neq 0\right)
$$

is necessarily isotropic with respect to the properly extended inner product $\langle$, (which we assume to be antilinear in the first argument and linear in the second argument). Consequently, we find

$$
\langle f, f\rangle=\left\langle f_{2}, f_{2}\right\rangle+\left\langle f_{3}, f_{3}\right\rangle=0 \text {. }
$$

By multiplying $f$ by a suitable unimodular number we can achieve that $\left\langle f_{2}, f_{3}\right\rangle=0$ and also that $f_{3}$ is time-like with positive third component. If $f_{1}$ is so chosen that $f_{1}$, $f_{2}, f_{3}$ is a Lorentz basis then $f_{1}$ is a space-like eigenvector of $S$ to a real eigenvalue which we denote by $a_{1}$. In short, we see that there exists a matrix $U \in \operatorname{SU}(1,1)$ such that

$$
O^{T}(U) \mathfrak{A} O(U)=\left(\begin{array}{rrr}
-a_{1} & 0 & 0 \\
0 & c & a \\
0 & a & -c
\end{array}\right), \quad a>0
$$

Actually, by means of a transformation of type (1.26) we can always achieve $c=0$. In the sequel we will assume that $\mathfrak{A}$ has already the form (15) with $c=0$. 
The functions (5.3), (5.4), and (2.10) take the form

$$
\begin{aligned}
& \boldsymbol{e}(\lambda)=\left(\frac{b_{1}}{\lambda-a_{1}}, \frac{\lambda b_{2}-a b_{3}}{\lambda^{2}+a^{2}}, \frac{a b_{2}+b_{3}}{\lambda^{2}+a^{2}}\right), \\
& g(\lambda)=1+\frac{b_{1}^{2}}{\left(\lambda-a_{1}\right)^{2}}+R(\lambda), \text { where } \quad R(\lambda)=\operatorname{Re}\left[\frac{b^{2}}{(\lambda-i a)^{2}}\right]
\end{aligned}
$$

and $b=b_{2}+i b_{3}$,

$$
\begin{aligned}
\Delta(\boldsymbol{e})= & -\left(\lambda^{2}+a^{2}\right) e_{1}^{2}-\left(\lambda-a_{1}\right) \operatorname{Re}\left[(\lambda+i a)^{2}\left(e_{2}+i e_{3}\right)^{2}\right] \\
& -\left(\lambda^{2}+a^{2}\right) e_{1}^{2} \text { for } \lambda=a_{1} \\
& \cdot \frac{1}{2}\left(\lambda-a_{1}\right)\left(\lambda^{2}+a^{2}\right) g^{\prime}(\lambda) \text { for } \lambda \neq a_{1} .
\end{aligned}
$$

If $b_{1} \neq 0$ there are no c. p. of the second kind, that is to say, all c. p. arise from (simple) zeros of $g(\lambda)$. This function has between zero and three pairs of zeros, each pair giving rise to an elliptic-hyperbolic pair of c.p. (Of course, a pair of zeros may coalesce giving rise to a degenerate c.p.) In any case, the total index of all c.p. equals zero.

This last property still holds true if $b_{1}=0$, although in this case the hyperbolic [see (18)] pair of c.p. of the second kind

$$
f_{1}^{ \pm}=\left( \pm\left[-g\left(a_{1}\right)^{1 / 2}\right], \frac{a_{1} b_{2}-a b_{3}}{\lambda^{2}+a^{2}}, \frac{a b_{2}+a_{1} b_{3}}{\lambda^{2}+a^{2}}\right)
$$

will appear as soon as also $g\left(a_{1}\right)<0$. But it is easy to see that its contribution of -2 to the total index is cancelled by a pair of elliptic c.p. of the first kind corresponding to zeros of $g(\lambda)$ that necessarily will appear on different sides of $a_{1}$.

Actually, the Case $\mathrm{II}^{\mathrm{C}}, b_{1}=0$ can be treated in much more detail. Indeed, in this case it follows from (17) that $g(\lambda)=1+R(\lambda)$. We will show that $R(\lambda)$ has either one or two relative minima depending on whether $\boldsymbol{b}$ is time-like or space-like. In the first case there are therefore maximal 2 pairs, in the second case maximal one pair of c.p. of the first kind. An explicit determination of these minima will therefore allow us to establish precisely how many pairs of c.p. of the first kind are present.

First we determine a number $\psi$ in the interval $-\pi<\psi \leqq \pi$, so that

$$
b^{2}=B i e^{-i \varphi}, \quad B=b_{2}^{2}+b_{3}^{2} .
$$

In particular,

$$
b_{2}^{2}-b_{3}^{2}=-\langle\boldsymbol{b}, \boldsymbol{b}\rangle=B \sin \psi .
$$

If we also set $\lambda+i a=r e^{i \phi}(a>0)$ we find $\lambda=r \cos \phi=a \cot \phi$ and

$$
R(\lambda)=\frac{B}{r^{2}} \sin (\psi-2 \phi) .
$$

In order to find the relative extrema of $R(\lambda)$ we notice that

$$
R^{\prime}(\lambda)=-\frac{2 B}{r^{3}} \sin (\psi-3 \phi) .
$$


Generically, there are three extrema corresponding to the three angles $\phi_{k}=\frac{\psi}{3}-k \frac{\pi}{3}(k=-1,0,1)$. They are located at the positions $\lambda_{k}=a \cot \phi_{k}$ $(k=-1,0,1)$ and a short calculation yields

$$
R\left(\lambda_{k}\right)=\frac{B}{a^{2}} \sin ^{3}\left(\frac{\psi}{3}+\frac{2 \pi}{3} k\right), k=-1,0,1 .
$$

The relative order of the numbers $R\left(\lambda_{k}\right)(k=-1,0,1)$ is obviously the same as the relative order of the imaginary parts of the unimodular numbers exp $i\left(\frac{\psi}{3}+\frac{2 \pi}{3} k\right)$. Using the geometrical fact that the latter numbers form the vertices of an equilateral triangle we establish the following order relation between the extrema

$$
\begin{aligned}
-\pi<\psi \leqq-\frac{\pi}{2}: & R\left(\lambda_{0}\right) \leqq R\left(\lambda_{-1}\right)<0<R\left(\lambda_{1}\right) \\
-\frac{\pi}{2}<\psi \leqq 0: & R\left(\lambda_{-1}\right)<R\left(\lambda_{0}\right) \leqq 0<R\left(\lambda_{1}\right) \\
0<\psi \leqq \frac{\pi}{2}: & R\left(\lambda_{-1}\right)<0<R\left(\lambda_{0}\right) \leqq R\left(\lambda_{1}\right) \\
\frac{\pi}{2}<\psi \leqq \pi: & R\left(\lambda_{-1}\right)<0 \leqq R\left(\lambda_{1}\right)<R\left(\lambda_{0}\right) .
\end{aligned}
$$

If $-\pi<\psi<0, b$ is time-like and the number of pairs of c.p. of the first kind is 0,1 or 2 depending on whether none, one or two of the numbers $R\left(\lambda_{0}\right), R\left(\lambda_{-1}\right)$ is smaller than -1 . If $0 \leqq \psi \leqq \pi, \boldsymbol{b}$ is null- or space-like and a pair of c.p. of the first kind exists precisely if $R\left(\lambda_{-1}\right)+1<0$.

Notice that if both numbers $1+R\left(\lambda_{0}\right)$ and $1+R\left(\lambda_{-1}\right)$ are positive, there are no c.p. whatsoever. This means that under this condition (which, for example, is satisfied if $\boldsymbol{b}=0)$ the Hamiltonian $K^{(2)}(\boldsymbol{x})$ [defined in (5.1)] has no periodic solution whatsoever.

In the Case $\mathrm{II}^{\mathrm{C}}$ the origin of $R^{4}$ is always unstable. Indeed, remembering that $a>0$ we see that the corresponding Sokol'skii-function

$$
Q(\phi)=a_{1} \sin ^{2} \phi+2 a \sin \phi-a_{1}
$$

has always a simple zero $\phi_{0}$ given by a solution of the equation

$$
\sin \phi_{0}=\left\{\begin{array}{lll}
a_{1}^{-1}\left[\left(a^{2}+a_{1}^{2}\right)^{1 / 2}-a\right] & \text { if } & a_{1} \neq 0 \\
0 & \text { if } & a_{1}=0
\end{array}\right.
$$

Our contention now follows from Theorem 3.

\section{An Example}

In this section we illustrate some of the theoretical developments of Sections 4 and 5 with the help of the example Hamiltonian

$$
H=\frac{1}{2}\left[\left(x_{1}^{2}+y_{1}^{2}\right)-\left(x_{2}^{2}+y_{2}^{2}\right)\right]+x_{1}^{2} x_{2}+\mu x_{2}^{3} .
$$


Notice, that the third order term is the same as in the Henon-Heiles model [6]. The normal form is of the type (5.7) with

$$
\begin{array}{ll}
a_{1}=-a_{2}=2+\mu & a_{3}=\frac{1}{2}\left[15 \mu^{2}+12 \mu-1\right] \\
b_{1}=b_{2}=0 & b_{3}=\frac{15}{2}\left(\frac{1}{9}-\mu^{2}\right) .
\end{array}
$$

The pair of c.p. of the second kind

$$
f_{1}^{ \pm}=\left( \pm 2\left[-\frac{1}{3}\left(\mu+\frac{2}{3}\right)\right]^{1 / 2} /|\mu+1|, 0,\left(\mu+\frac{1}{3}\right) / \mu+1\right)
$$

exists for $\mu<-\frac{2}{3} \mu \neq-1$ with index

$$
\text { ind } \boldsymbol{f}_{1}^{ \pm}= \begin{cases}+1 & -2<\mu<-1 \\ -1 & \mu<-2,-1<\mu<-\frac{2}{3} .\end{cases}
$$

Similarly, the pair of c.p. of the second kind

$$
f_{2}^{ \pm}=\left(0, \pm\left[-\frac{28}{15}\left(\mu+\frac{2}{15}\right)\right]^{1 / 2} /\left|\mu+\frac{3}{5}\right|,\left(\mu-\frac{1}{3}\right) / \mu+\frac{3}{5}\right)
$$

exists for $\mu<-\frac{2}{15} \mu \neq-\frac{3}{5}$ with index

$$
\text { ind } \boldsymbol{f}_{2}^{ \pm}=\left\{\begin{array}{cc}
+1 & \mu<-2,-\frac{3}{5}<\mu<-\frac{1}{3} \\
-1 & -2<\mu<-\frac{3}{5},-\frac{1}{3}<\mu<-\frac{2}{15} .
\end{array}\right.
$$

The only c.p. of the first kind are $\pm \boldsymbol{k}$. They are present for all $\mu$ and we have

$$
\begin{aligned}
\text { ind } \boldsymbol{k} & = \begin{cases}+1 & |\mu|>\frac{1}{3} \\
-1 & |\mu|<\frac{1}{3},\end{cases} \\
\text { ind }(-\boldsymbol{k}) & = \begin{cases}+1 & \mu<-\frac{2}{3},-\frac{1}{3}<\mu<-\frac{2}{15}, \mu>\frac{1}{3} \\
-1 & -\frac{2}{3} \leqq \mu<-\frac{1}{3} ;-\frac{2}{15} \leqq \mu<\frac{1}{3} .\end{cases}
\end{aligned}
$$

Theorem 6 implies: the origin of $R^{4}$ is unstable for $-1<\mu<-\frac{3}{5}$ and $|\mu|<\frac{1}{3}$ and stable for $\mu$ lying in the interior of the complementary intervals.

Acknowledgement. The author would like to express his sincerest thanks to Dr. Robert Deck for a critical reading of this work and to Mrs. Kay Locke for her superb typing of the manuscript. The author is also indebted to the referee of the present paper for pointing out to him that the Case $\mathrm{II}^{\mathrm{C}}$ has been omitted in the original version of the manuscript.

\section{References}

1. Kummer, M.: On resonant non-linearly coupled oscillators with two equal frequencies. Commun. math. Phys. 48, 53-79 (1976)

2. Moser, J.K.: Regularization of Kepler's problem and the averaging method on a manifold. Commun. Pure Appl. Math. 23, 609-636 (1970)

3. Moser,J.K.: On invariant curves of area preserving mappings of the annulus. Nachr. Akad. Wiss. 1, 1-20 (1962). See also : Siegel, C.L., Moser,J.K. : Lectures on celestial mechanics. Berlin-HeidelbergNew York: Springer 1971

4. Sokol'skii, A.G. : On stability of an autonomous Hamiltonian system with two degrees of freedom in the case of equal frequencies. Appl. Math. Mech. 38, 791-799 (1974) 
5. Moser,J.K.: Lectures on Hamiltonian systems. Mem. Amer. Math. Soc. 81, 1-57 (1968)

6. Henon, M., Heiles, C.: The applicability of the third integral of motion; some numerical experiments. Astron. J. 69, 73-79 (1964)

7. Kummer, M.: An interaction of three resonant modes in a non-linear lattice. J. Math. Anal. Appl.52, 64-104 (1975)

8. Schmidt,D., Sweet,D. : A unifying theory in determining periodic families for Hamiltonian systems at resonance. J. Diff. Eq. 14, 597-609 (1973)

Communicated by J. Glimm

Received May 1, 1977 